Florida International University FIU Digital Commons

6-30-2014

\title{
Elevated Progesterone In Yolk As a Moderator of Prenatal and Postnatal Auditory Learning in Bobwhite Quail
}

Joshua A. Herrington

Florida International University, Joshuahvt@gmail.com

DOI: $10.25148 /$ etd.FI14071131

Follow this and additional works at: https://digitalcommons.fiu.edu/etd

\section{Recommended Citation}

Herrington, Joshua A., "Elevated Progesterone In Yolk As a Moderator of Prenatal and Postnatal Auditory Learning in Bobwhite Quail" (2014). FIU Electronic Theses and Dissertations. 1422.

https://digitalcommons.fiu.edu/etd/1422 


\title{
FLORIDA INTERNATIONAL UNIVERSITY \\ Miami, Florida
}

\section{ELEVATED PROGESTERONE IN YOLK AS A MODERATOR OF PRENATAL AND POSTNATAL AUDITORY LEARNING IN BOBWHITE QUAIL}

\author{
A dissertation submitted in partial fulfillment of the \\ Requirements for the degree of \\ DOCTOR OF PHILOSOPHY \\ in \\ PSYCHOLOGY \\ By
}

Joshua A. Herrington

2014 
To: Interim Dean Michael R. Heithaus

College of Arts and Sciences

This dissertation, written by Joshua A. Herrington, and entitled Elevated Progesterone in Yolk as a Moderator of Prenatal and Postnatal Auditory Learning in Bobwhite Quail, having been approved in respect to style and intellectual content, is referred to you for judgment.

We have read this dissertation and recommend that it be approved

$\begin{array}{r}\hline \text { John Berry } \\ \hline \text { Bethany Reeb-Sutherland } \\ \hline \text { Anthony Dick } \\ \hline \text { Robert Lickliter, Major Professor }\end{array}$

Date of Defense: June 09, 2014

The dissertation of Joshua A. Herrington is approved.

Interim Dean Michael R. Heithaus

College of Arts and Sciences

Dean Lakshmi N. Reddi

University Graduate School

Florida International University, 2014 


\section{DEDICATION}

I dedicate this dissertation to my family; especially my wife, Raven, and my mother, Mary. Their support and love made it possible to chip at the mountain until I emerged with joy and wonder on the other side. 


\section{ACKNOWLEDGMENTS}

I wish to thank the members of my committee for all of their support, guidance, and especially their boundless patience as I completed this dissertation. Dr. John Berry was extremely generous in his allowance of lab space and insight into the methodology for hormone extraction and analysis, without which this dissertation would not have been possible. Most of all, I would like to thank my major professor, Dr. Robert Lickliter, for his invaluable years of mentorship.

I would also like to thank my lab associates at the developmental psychobiology lab, the quail crew, for their opinions and good humor. 


\section{ABSTRACT OF THE DISSERTATION \\ ELEVATED PROGESTERONE IN YOLK AS A MODERATOR OF PRENATAL AND POSTNATAL AUDITORY LEARNING IN BOBWHITE QUAIL By}

Joshua A. Herrington

Florida International University, 2014

Miami, Florida

Professor Robert Lickliter, Major Professor

Recent studies have established that yolk hormones of maternal origin have significant effects on the physiology and behavior of offspring in birds. Herrington (2012) demonstrated that an elevation of progesterone in yolk elevates emotional reactivity in bobwhite quail neonates. Chicks that hatched from progesterone treated eggs displayed increased latency in tonic immobility and did not emerge as quickly from a covered location into an open field compared to control groups. For the present study, three experimental groups were formed: chicks hatched from eggs with artificially elevated progesterone $(\mathrm{P})$, chicks hatched from an oil-vehicle control group (V), and chicks hatched from a non-manipulated control group (C). Experiment 1 examined levels of progesterone with High Performance Liquid Chromatography/tandem Mass Spectroscopy (HPLC/MS) from prenatal day 1 to prenatal day 17 in bobwhite quail egg yolk. In Experiment 2, bobwhite quail embryos were passively exposed to an individual maternal assembly call for 24 hours prior to hatching. Chicks were then tested individually for their preference between the familiarized call and a novel call at 24 and 48 hours following hatching. For Experiment 3, newly hatched chicks were exposed to an 
individual maternal assembly call for 24-hrs. Chicks were then tested for their preference for the familiarized call at 24 and 48-hrs after hatch. Results of Experiment 1 showed that yolk progesterone levels were significantly elevated in treated eggs and were present in the egg yolk longer into prenatal development than the two control groups. Results from Experiment 2 indicated that chicks from the $\mathrm{P}$ group failed to demonstrate a preference for the familiar bobwhite maternal assembly call at 24 or 48-hrs after hatch following 24hrs of prenatal exposure. In contrast, chicks from the $\mathrm{C}$ and $\mathrm{V}$ groups demonstrated a significant preference for the familiarized call. In Experiment 3, chicks from the P group showed an enhanced preference for the familiarized bobwhite maternal call compared to chicks from the $\mathrm{C}$ and $\mathrm{V}$ groups at 24 and 48-hrs after hatch. The results of these experiments suggest that elevated maternal yolk hormone levels in pre-incubated bobwhite quail eggs can influence auditory perceptual learning in embryos and neonates. 


\section{TABLE OF CONTENTS}

\section{CHAPTER}

PAGE

I. GENERAL INTRODUCTION 1

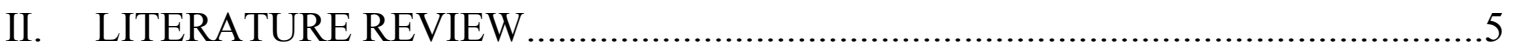

Influence of prenatal hormones on mammalian neonatal behavior .............................5

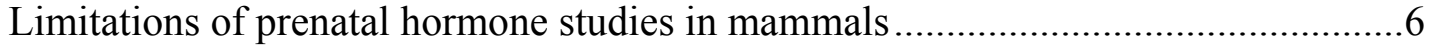

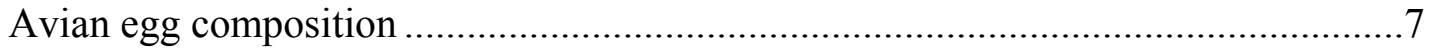

Presence and distribution of hormones in avian eggs ...................................... 8

III. THE TRANSFER OFMATERNALLY DERIVED HORMONES INTO

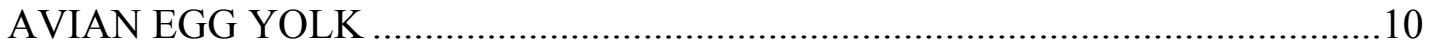

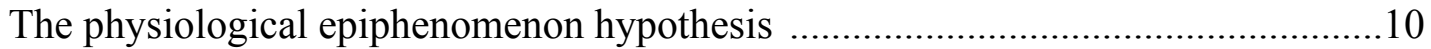

The flexible distribution hypothesis............................................................. 12

No relationship between maternal experience and egg steroid composition.............12

Behavioral effects of elevated glucocorticoid exposure in birds .............................13

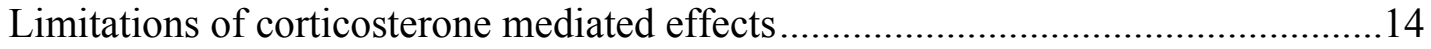

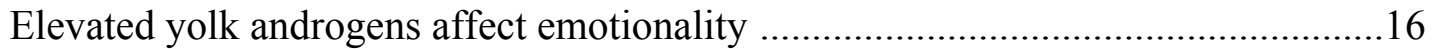

Elevated yolk androgens affect prenatal and postnatal perception...........................18

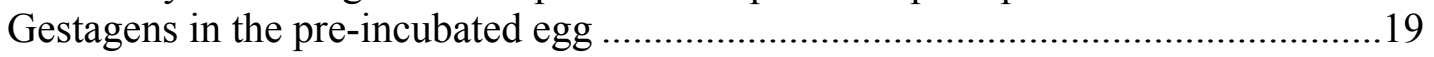

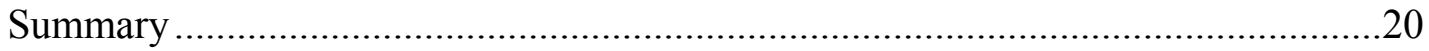

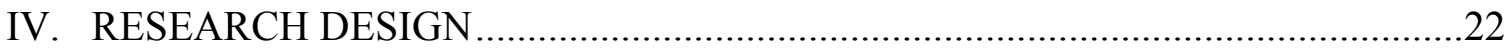

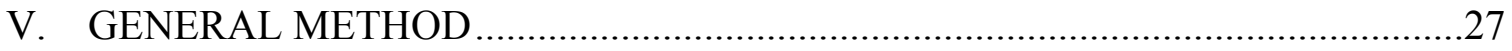

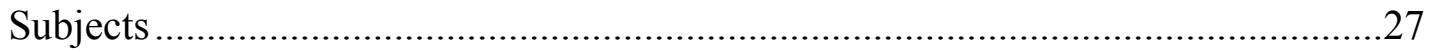

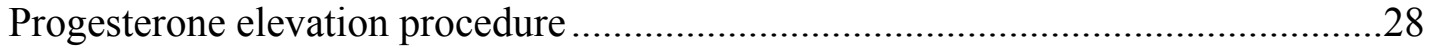

Analysis of progesterone in egg yolk/embryonic growth ..................................28

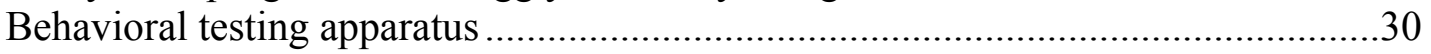

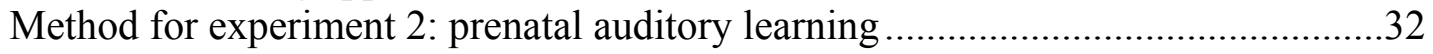

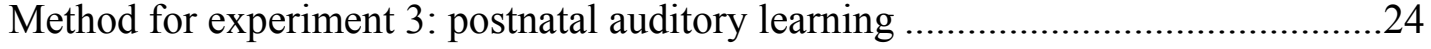

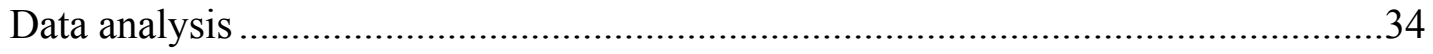

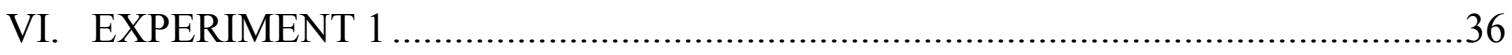

Hormone content and growth in prenatal development .......................................36

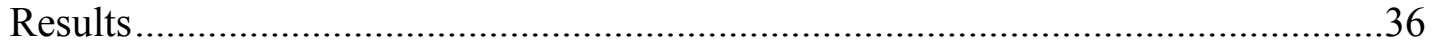

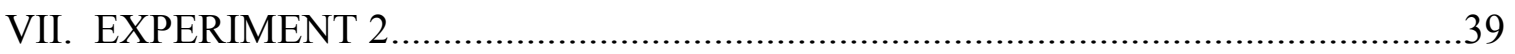

Effects of prenatal progesterone elevation on prenatal auditory learning .................39

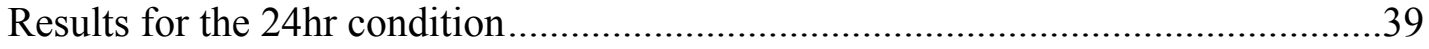

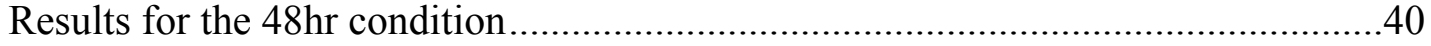


Summary

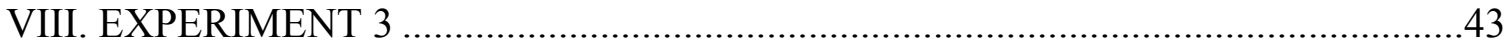

Effects of prenatal progesterone elevation on postnatal auditory learning.................43

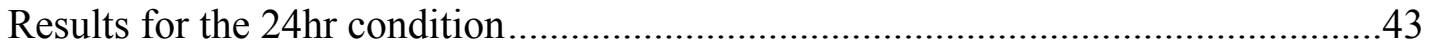

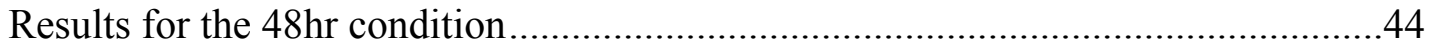

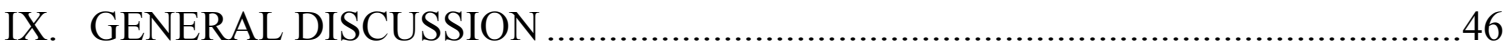

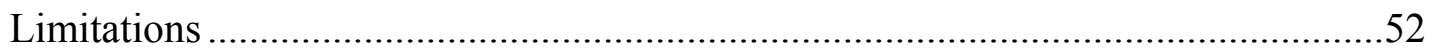

Complexity of non-genetic prenatal influences .....................................................53

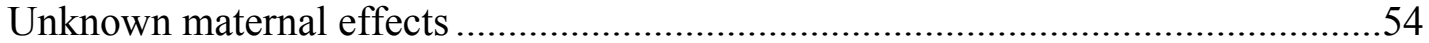

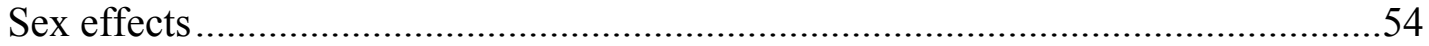

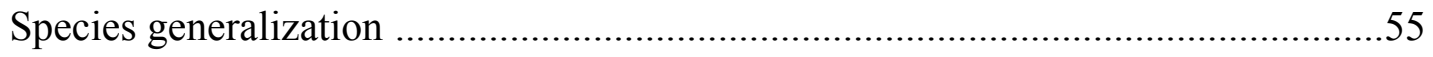

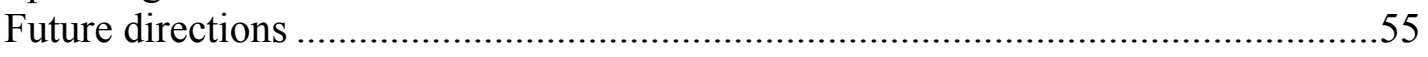

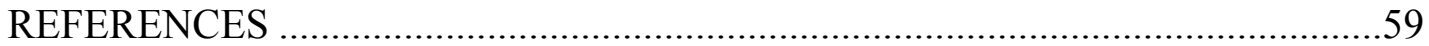

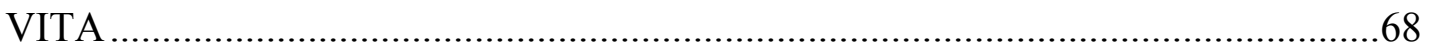




\section{CHAPTER I}

\section{GENERAL INTRODUCTION}

\section{Introduction}

Both similarities and inter-individual difference in phenotypic traits are present across individuals within a species. Inter-individual variations in phenotypes were long thought to be the result of an individual's genetic code which was modified over thousands, hundreds of thousands, or millions of years due to chance mutation and/or the process of natural selection. Conceptualizing the gene as the sole determinant of phenotypic traits was commonplace across the life sciences for much of the past century (Honeycutt, 2006; Lickliter \& Honeycutt, 2003).

Advances in the fields of genetics, epigenetics, and embryology suggest that interindividual differences in phenotypes emerge via a series of complex interactions across development between the individual and biological and non-biological influences that constitute the environment in which the organism is embedded (Lickliter \& Honeycutt, 2003). Biological influences in the environment include conspecifics and parents, and both can influence the development of an individual's behavior. A growing body of evidence suggests that influences from mothers and fathers, collectively known as "parental influences", play a fundamental role in the development of an individual's phenotype (Mousseau \&Fox, 1998).

Studies have shown that natural variations in non-biological environmental conditions and parent biology can affect parent/offspring interactions, which can indirectly affect the phenotype of their offspring. In other words, certain environmental conditions can alter parental behaviors, which can in turn alter phenotypes in their 
offspring. As an example, bird parents that rear their offspring in a food-rich environment provide better nutrition compared to parents who raise offspring in a foodpoor environment, thus indirectly affecting their offspring's physical growth (Groothuis \& Schwabl, 2008). In addition, individual differences in parental biology can also affect offspring phenotypes. For example, variations in the expression of glucocorticoid stress hormone levels can alter foraging behavior in free-ranging populations of birds, which can affect nutrition levels and lead to variations in the physiology and stress related behaviors of their offspring (Wingfield \& Kitaysky, 2002).

A wealth of studies suggest that maternal behaviors can act as a strong influence on the emergence of offspring phenotype, altering behavioral and physiological outcomes across development (Mousseau \& Fox, 1998). For example, studies have shown that maternal behaviors during the early postnatal period in rodents can significantly alter behavior across the lifespan of their offspring(Francis, \& Meaney, 1999; Roth, Lubin, Funk, \& Sweatt, 2009). Variation in patterns of licking and grooming behaviors of rat dams result in specific changes to their offspring's phenotype (Plotsky \& Meaney, 1993). Rat pups reared by mothers with low levels of licking and grooming behaviors (LLG) exhibit elevated fear and stress responses in adulthood compared to offspring of mothers with high levels of licking and grooming behaviors (HLG) (Champagne, Francis, Mar, \& Meaney, 2003). Rat pups that were reared with HLG mothers displayed a decreased hypothalamic-pituitary-adrenal (HPA) response to stressful stimuli compared to pups that were reared with LLG mothers (a et al., 2004). These changes in fear- and stress-related behaviors elicited by the mother's HLG behaviors during infancy have been shown to persist into adulthood. Maternal activities of young females that experience low 
frequencies of LLG behaviors as pups are similar to that of their mother's (Francis, Diorio, Liu, \& Meaney, 1999). Experiments using cross-fostering techniques confirmed the non-genetic nature of the maternal influences, as behavioral outcomes in pups birthed from LLG mothers that were raised with high licking/grooming (HLG) mothers were the same as pups reared by HLG mothers (and vice versa) (Francis, Champagne, Liu, \& Meaney, 1999). These studies in rats demonstrate that maternal behaviors during neonatal development can directly influence phenotype without appeals to genetic determinism, suggesting the possibility of other maternally-derived influences on postnatal behavior. Although the majority of studies that have demonstrated the effect of maternal behavior on offspring phenotype have been in mammals, there are studies in birds that have found maternal influences on chicks' behavior. Studies with domestic pullets found that hatchlings reared in the presence of brood hens resulted in modified behavioral hierarchies and aggressive behaviors compared to chicks that were not reared with brood hens (Perre, Wauters, \& Richard-Yris, 2002). A similar study demonstrated that domestic chicks reared with a brood hen displayed fewer freezing behaviors (a classic fear-related behavior in birds) compared to domestic chicks that had been raised with a heat lamp only (Shimmura, Kamimura, Azuma, Kansaku, Uetake, \& Tanaka, 2010). In Japanese quail, the presence of a hen during the first few days after hatching significantly alters hatchling's emotional reactivity; both motivation to return to brood-mates and emotional reactivity are higher in individuals raised with mothers compared to individuals raised without mothers (Bertin \& Richard-Yris, 2005). In addition, the behavioral characteristics of mother quail hens have been found to influence offspring behavior. Young Japanese quail raised by mothers that display high levels of emotional 
reactivity exhibit high levels of emotional reactivity themselves, compared to chicks raised with mothers that display low levels of emotional reactivity (Richard-Yris, Michel, $\&$ Bertin, 2005).

Maternal influences on offspring behavior are not limited to the period after birth or hatching. There is also a great deal of influence provided by mothers during prenatal development that can affect offspring's postnatal behavior. For example, studies in bobwhite quail hatchlings have suggested that hormones of maternal origin present in the prenatal environment can affect prenatal auditory learning and postnatal emotional reactivity (Bertin, Richard-Yris, Mostl, \& Lickliter, 2009; Herrington \& Lickliter, 2012). My review of the literature examines what is currently known about maternal hormonal influences in the prenatal environment, and how these influences can affect the postnatal behaviors of their offspring. Particular attention is paid to the influence of prenatal hormone exposure on neonatal behavior in birds and mammals. I separate findings between the two, as mammals and birds undergo prenatal development in very different environments. 


\section{CHAPTER II}

\section{LITERATURE REVIEW}

In addition to maternal influences on offspring behavior in the neonatal period, there are many maternal influences present in the prenatal environment (e.g. chemical, tactile, and auditory stimuli) that guide and constrain the experiences of the developing organism. Over the course of embryonic and fetal development, an organism is exposed to not only sensory information from its environment, but also an array of hormones and chemicals that can affect postnatal behavior (Alberts \& Cramer, 1988).

Several studies have found evidence that maternal hormones are present in the prenatal environment of mammals and birds, and can affect postnatal phenotypes. Specifically, the alteration of hormones of maternal origin like testosterone (Sarma, Manikkam, Herkimer, Dell'Oroco, Welch, Foster \& Padmanabhan, 2005) and cortisol (Holms \& Seckl, 2007) in the prenatal environment have been shown to lead to physiological and behavioral changes that persist across the lifespan in mammals. Influence of prenatal hormones on mammalian neonatal behavior

Differential exposure to exogenous sex hormones in the prenatal environment can direct sex-specific differences in behavior after birth in mammals. Hormones that circulate in a pregnant female's bloodstream are metabolized by the placenta, and thereby typically block some maternal hormone exposure to the fetus (Chan, Vasilopoulou, \& Kilby, 2009). Regardless, some hormones of maternal origin can pass through or be produced by the placenta and make their way into the bloodstream of a developing fetus, which can influence the emergence of postnatal phenotypes, such as the masculinization of female spotted hyenas via testosterone exposure in utero (Licht, Frank, Pavgi, 
Yalcinkaya, Siiteri, \& Glickman, 1992). Mature spotted hyena females develop a pseudo-penis, display increased aggressive behavioral tendencies, and can dominate males in their social groups (Licht et al, 1992). It is presumed that spotted hyena females are exposed to, among other things, high circulating levels of androstenedione in utero, which may account for their masculine phenotype.

Mammals, which develop in a uterine environment, can be exposed to hormones from a variety of sources other than the mother. For example, female gerbils that are positioned between two males in the uterus of their mother are exposed to higher levels of testosterone than a female positioned next to two other females. Subsequently, female gerbils that are exposed to higher levels of prenatal testosterone produced by their neighboring male siblings display increased testosterone levels and an overall more masculine phenotype across their lifespan (Clark and Galef, 1995). The effects of androgens produced by neighboring siblings in utero have been discovered in other mammalian species as well. Female mice positioned between two males may appear less sexually attractive, present a lower sexual receptivity, and display higher aggressiveness than females placed between two other females (vom Saal, 1989). Studies such as these indicate that hormones other than those of maternal origin may also influence growth and development in mammalian species.

Limitations of prenatal hormone studies in mammals

There are several methodological challenges inherent in studies of mammals to examine the specific effects of hormones of maternal origin that are present during prenatal development. Highly controlled experimental manipulation of hormone concentrations in the uterus is difficult in mammals, as the embryo develops within its 
mother's body and is thus exposed to maternal hormone production, as well as exogenous hormones from possible neighboring siblings. Exact measurements of the quality and quantity of maternal hormones that are passed to the fetus via the placenta are difficult to obtain, as environmental interactions which affect maternal behavior and hormone expression vary widely in mammals. Further complications include the inherent difficulty of obtaining in-vivo samples of hormones in the uterine environment. Persistent blood and/or amniotic samples are a necessary component for the study of maternal hormone influence in prenatal development; however this method of data collection can cause both discomfort and provides a confounding variable as a result of potential activation of a stress response in the mother.

\section{Avian egg composition}

Albumen and yolk of avian eggs contain a variety of compounds. In the albumen, or egg white, which is mostly composed of water (roughly $90 \%$ ) there are various vitamins, salts and proteins. It also contains lysozymes to provide protection from bacteria (Sauveur 1988; Saino et al. 2002), and may include glucocorticoid stress hormones like corticosterone (Love et al., 2008). Corticosterone, like cortisol in mammals, is released from the adrenal glands after activation of the hypothalamicpituitary-adrenal axis and sympathetic nervous response (McEwen \& Sapolsky, 1995).

The yolk is mostly comprised of lipids (around 30\%), and proteins of various kinds (approximately 16\%), as well as minerals, vitamins, and a small amount of water. Carotenoids can also be found in yolk, and are comprised mainly of xanthophylls, lutein, and zeaxanthin (antioxidant molecules) that are ingested as part of maternal diet. Variation of yolk caratinoids contributes to color variations (from light yellow to deep 
orange). Interestingly, high testosterone levels in the yolk of domestic chickens have been linked with a decrease in carotenoid levels (Royle, Surai, \& Hartley, 2001).

Avian eggs offer a controlled environment to study the effects of maternally derived hormones and their relationship to other compounds found in egg yolk that may influence the growth and development of their offspring across their lifespan. Groothuis and Schwabel (2007) argue that the use of an avian model to study maternally-derived hormone effects is ideal, in that bird embryos develop in an egg environment that is much more sequestered than that of a mammal's uterus in terms of their exposure to endogenous hormones. In addition, the large eggs of birds offer the opportunity to experimentally manipulate yolk hormone levels and study the effects on individual behavior and physiology with relative ease.

Presence and distribution of hormones in avian eggs

Earlier studies with birds found that testosterone, a steroid hormone, varies between clutches of canary (Serinus canaria) and zebra finch (Poephila guttata) eggs (Schwabl, 1993). Further studies in birds resulted in the discovery that hormonal variation between clutches existed in the yolk of eggs of the same species (Groothuis \& Schwabl, 2002). Analysis of the yolk and albumin of several species of birds revealed that eggs contain substantial concentrations of androgens, as well as other hormones of maternal origin that are essential for development (Gil, 2003; Romanoff \& Romanoff, 1949). Furthermore, these hormones are distributed differentially in different layers of yolk.

In birds, the ovary produces sexual steroids that can be transferred to a developing egg. These steroids are produced in the different follicle cell layers that encapsulate the 
oocyte. For example, progesterone is produced by granulosa cells, which is used by theca interna cells to produce androgens such as testosterone and androstenedion via side chain cleavage. These androgens are then metabolized into oestrogens by the theca externa cells (Huang et al. 1979; Porter et al. 1989). It is currently unknown whether the hormones that circulate in the hen's bloodstream are actively transferred into the egg yolk, or are found there by passive diffusion (Groothuis \& Schwabl, 2008).

Analysis of avian egg yolks have found that androgens and glucocorticoids are present in the preincubated egg for the first 2-3 embryonic days; however after prenatal day 3 , steroid hormone levels drastically decrease via some unknown mechanism (Groothuis \& Schwabl, 2008). As the embryo develops, androgen and glucocorticoid hormone levels gradually increase. The mechanism that underlies the decreased concentration of maternal hormones in bird egg yolk after the first few days of prenatal development is currently unknown and under investigation. 


\section{CHAPTER III}

\section{THE TRANSFER OF MATERNALLY DERIVED HORMONES INTO AVIAN EGG}

\section{YOLK}

Unlike mammals, avian females can influence their embryo's prenatal hormone exposure only once, during the formation of the egg. While the egg is being formed in the reproductive tract of female birds, theca and granulosa cells that surround the oocyte produce hormones that end up in different layers of egg yolk (Gil, 2003). Maternal yolk hormone deposits during egg formation are influenced by a variety of environmental conditions; including their physical environment (Schwabl, 1996; Guesdon et al., 2011; Guibert et al., 2010), sexual and social context (Gil, 2008), social status (Tanvez, Parisot, Chastel, \& Leboucher, 2008), body condition (Verboven et al., 2003), age (Okuliarova, Skrobanek, \& Zeman, 2009), and laying order in a clutch (Gil, 2008).

The mechanism by which hormones from mother birds are transferred to offspring is unknown. Currently, there are three hypotheses that attempt to explain how behavioral traits of the mother are mirrored in the hormone concentration of her eggs. The competing hypotheses were created by Groothuis and Shwable (2008) after they reviewed the existing literature on the presence of maternally derived hormones in bird eggs.

\section{The physiological epiphenomenon hypothesis}

The Physiological Epiphenomenon Hypothesis (PEH) predicts a positive relationship between the blood hormone levels in a laying female and the hormones that are deposited in the yolk of their eggs (Groothuis \& Schwabl, 2008). For example; famine (Kitaysky, Piatt, \& Wingfield, 2007), high parasite load (Bruener \& Hahn, 2003), or high predator 
density (Silverin, 1998) could all contribute to higher levels of circulating stress hormones, such as glucocorticoids, in a laying female's bloodstream (Groothuis \& Schwabl, 2008; Gil, 2003). The possible outcomes for this mechanism of yolk steroid deposits by the mother can be maladaptive, neutral, or adaptive to both her and her offspring (Groothuis \& Schwabl, 2008). The PEH specifically predicts that if there is high circulation of glucocorticoids and androgens in the circulatory system of a pregnant female bird, then there will be elevated amounts of these hormones diffusing into the egg yolk. The mechanism by which environmental influences affect the production and distribution of maternal hormones in avian eggs is displayed below in Figure 1.

Figure 1

Distribution of Hormones in Avian Eggs

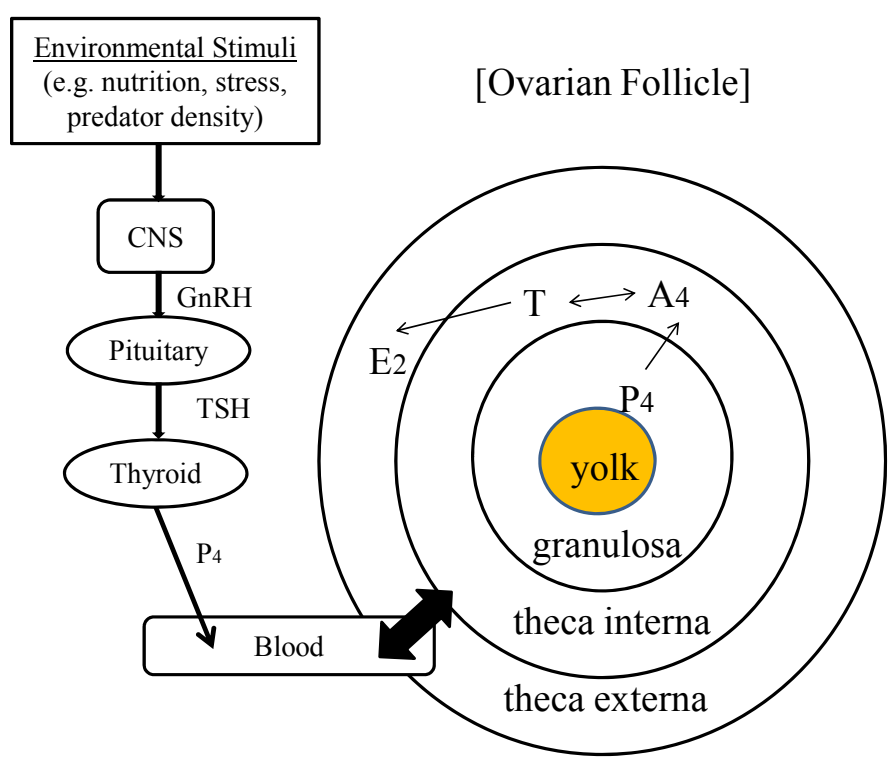

Fig 1: Graphic representation of steroidogenesis of a female bird. Adopted from Groothuis \& Schwabl, 2008. (P4: Progesterone; A4: Androstenedione; T: Testosterone; E2: Oestradiol). The arrows represent the pathway by which androgens are produced via progesterone acting as a precursor hormone. 


\section{The flexible distribution hypothesis}

The second hypothesis put forth by Groothuis and Crews (2008) is the Flexible Distribution Hypothesis (FDH), which predicts a negative relationship between maternal hormone circulation and the concentration of hormones found in the yolk of oocytes. Groothuis and Schwabl (2008) assume that the production of hormones comes at a cost to energy and fitness in female birds. In the FDH framework, a female bird's individual fitness would be lessened by increasing the amount of high hormone concentration into the egg, while at the same time providing an adaptive advantage to her offspring. No relationship between maternal experience and egg steroid composition

Groothuis and Schwabl (2008) also hypothesize a third possibility: that the transfer of hormones from the mother to the egg may not have any correlation or relationship. As such, there would be no relationship between the circulating levels of glucocorticoids or androgens found in the bloodstream of a female bird and the presence of higher or lower levels of glucocorticoids or androgens in the yolk of her eggs.

There is evidence to support all three of the hypotheses proposed by Groothuis and Schwabl (2008) by which females transfer hormones into the yolk of their offspring. Interestingly, the correlations between maternal testosterone concentration and the amount of testosterone deposited in her eggs prior to incubation can vary between species and populations of birds (see Table 1). 


\section{Table 1}

\section{Distribution of Hormones in Egg Yolk}

\begin{tabular}{lllll} 
correlation & hormone & species & reference & sample \\
\hline+ & $\mathrm{T}$ & Domestic canary & Schwabl (1996a) & Indv. females \\
+ & $\mathrm{T}$ & House finch & Badyaev et. al (2005) & Indv. females \\
0 & $\mathrm{~T}$ & House Sparrow & Egbert (2006) & Indv. females \\
0 & $\mathrm{~T}$ & Domestic canary & Marshall et al. (2005) & Group level \\
0 & $\mathrm{~T}$ & Eurasian starling & Pilz et al. (2003) & Group level \\
- & $\mathrm{T}$ & House sparrow & Mazuk et al. (2003) & Group level \\
- & $\mathrm{T}$ & Eastern bluebird & Navara et al. (2006) & Group level
\end{tabular}

Table 1: Correlation of maternal hormones in egg yolk (adopted from Groothuis et al., 2008). T represents maternally derived testosterone. The table represents a positive (+), negative (-), or no (0) correlation of maternal hormones to those found in the egg yolk of unincubated eggs.

\section{Behavioral effects of elevated glucocorticoid exposure in birds}

Although it is unclear whether the transfer of hormones from a hen to her eggs is a passive transfer, or if there are unknown compensatory or environmental mechanisms that control the transfer of hormones (Gil, 2003), there is evidence in the literature that hormones of maternal origin can affect behavioral outcomes soon after hatch, as well as across development into adulthood.

All bird species have a hypothalamic-pituitary-adrenal (HPA) axis. The HPA axis is comprised of structures within the subcortex (hypothalamus and pituitary) and the adrenal glands, which are located on the kidneys. The HPA axis acts as a bridge between the brain and the body when the "fight or flight" stress response is activated. 
Stress responses via activation of the sympathetic nervous system is regulated by the HPA axis by the release of hormones and neurotransmitters that act as precursors to hormone production. When an animal experiences a stressful stimuli, the hypothalamus releases corticotropin releasing hormone $(\mathrm{CRH})$ which signals the anterior pituitary to release adrenocorticotropic hormone (ACTH). The ACTH travels to the adrenal cortex, which then produces the glucocorticoid hormone corticosterone. The corticosterone then travels back to the hypothalamus signal the stop of CRH production, thus creating a negative feedback loop.

In line with the PEH (Groothuis \& Schwabl, 2008), a laying hen who experiences high levels of stress will produce high levels of circulating corticosterone during the formation of their eggs. Levels of corticosterone in the circulatory system of a female bird are positively related to the amount of stress those individual females experience. Researchers have found evidence of this by repetitive handling of laying Japanese quail to activate the HPA axis, and thereby induce the release of high levels of corticosterone (Hayward et al., 2006). Quail chicks who developed from mothers who had been repeatedly handled at the time of oogenesis (the time period where formation of the egg inside the mother's body occurs) displayed increased levels of HPA activation compared to chicks from hens that had not been repeatedly handled.

Direct manipulation of circulating glucocorticoids in laying Japaneese quail hens resulted in higher levels of corticosterone in the yolk compared to controls (Hayward \& Wingfield, 2004). In addition, quail chicks that had been hatched from eggs with experimentally elevated corticosterone levels displayed different physiological and behavioral changes (i.e., decreased birthweight and increased emotional reactivity) when 
compared to chicks from non-manipulated eggs (Hayward, Richardson, Grogan, \& Wingfield, 2006).

\section{Limitations of corticosterone mediated effects}

It is still under debate whether an increase of circulating corticosterone in the mother translates to higher concentrations of corticosterone in the egg. Researchers have previously implemented sensitive measures of yolk corticosterone after administration of adrenocorticotropic hormone (ACTH) to hens (Rettenbacher, Mostl, Hackl, \& Palme, 2005). Radiolabled corticosterone that was injected into laying female Japanese quail was not found in homogenized egg yolk (Rettenbacher et al. 2005). Rettenbacher et al. (2005) concluded that maternal hormones in avian species may not include the active transfer of circulating glucocorticoids from the laying hens to their eggs. The findings from the Rettenbacher et al. (2005) study suggests that glucocorticoids may not be found in high enough levels in the pre-incubated egg to affect prenatal and early postnatal behavior in their offspring. However, it was recently found that in captive barn owls, experimental elevation of maternal corticosterone within the normal physiological range translated into elevated concentrations of corticosterone in their eggs (Almasi, Rettenbacher, Muller, Brill, Wagner, \& Jenni, 2012).

There are potential mechanisms that may explain the differential outcomes that are inherent in studies of phenotypic variation via elevated maternally-derived glucocorticoid exposure in bird and avian embryos. The enzyme 11/-HSD2, which inactivates glucocorticoid processes in the placenta of mammals, has been found in the ovary of zebra finches (Katz, Oyama, Feng, chen, \& Schlinger, 2010) and in the gonads and oviduct of chickens (Henriksen, Rettenbacher, \& Groothuis, 2011; Klusoňová, 
Kučka, Ergang, Mikšík, Bryndová, \& Pácha, 2008). These findings might explain the low transfer rate of corticosterone in some studies from the avian females' plasma to her eggs. The presence of 11ß-HSD2 in birds and mammals suggest that the two species may have similar strategies to protect the embryo from overexposure to glucocorticoids of maternal origin.

\section{Elevated yolk androgens affect emotionality}

In contrast to corticosteroids produced by laying hens, which may or may not be found in yolk, androgens are present in pre-incubated eggs. Furthermore, elevated androgen concentrations in the pre-incubated egg yolk have been found to produce physiological and behavioral changes in several species of birds (Bertin et al., 2009, Sockman \& Schwabl, 2000). For example, Sockman \& Schwabl (2000) examined the specific effects of elevated pre-incubation androgen levels in the yolk in the American kestral. They found that prenatal elevation of androgen steroids in American kestral embryos resulted in nestlings that had higher corticosterone concentrations after birth (Sockman \& Schwabl, 2000). The elevated corticosterone levels in the kestral hatchlings affected their fitness in a negative way, as their survival rates were less than chicks that had not experienced elevated androgen levels during prenatal development.

A study in Japanese quail produced similar results (Daisley et al., 2005). Chicks with experimentally elevated androgen levels displayed higher levels of emotional reactivity and thus higher levels of circulating corticosterone regardless of sex (Daisley et al., 2005). High emotional reactivity behavior in birds is typically assessed by a higher incidence of freezing behavior or immobility in an open field, longer durations of tonic 
immobility, and/or fear of novel stimuli or response to restraint (Mignon-Grasteau et al., 2003).

Elevation of egg yolk steroid hormone content via injections are not the only way that levels of maternally derived hormones can be experimentally manipulated. One recent study found that a heat elevation challenge resulted in an elevation of progesterone and testosterone in pre-incubated chicken eggs (Bertin et al., 2013). In the same study, eggs laid by hens that did not experience a heat challenge produced eggs with less progesterone and testosterone compared to hens that had experienced elevated heat levels during oogenesis. An earlier study in Japanese quail indirectly manipulated levels of progesterone and androgens in pre-incubated eggs by exposure of laying hens to human handling (Bertin et al., 2008). Japanese quail hens that were exposed to repeated human handling produced eggs with less progesterone and elevated androgen levels compared to hens that did not receive repeated human handling (Bertin et al., 2008). Chicks that hatched from eggs laid by mothers who had been repeatedly handled experienced elevated emotional reactivity compared to chicks hatched from eggs laid by hens who had not been handled. The results from the Bertin et al. (2008) study were not reproduced in Herrington (2012), as birds that had elevated progesterone levels in the egg yolk before incubation produced chicks that were more emotionally reactive compared to chicks that did not receive progesterone elevation via egg injection.

A study with bobwhite quail embryos found that elevating yolk testosterone levels prior to incubation resulted in a modification of emotional reactivity (Bertin et al., 2009). In a series of experiments, bobwhite quail that had elevated levels of prenatal testosterone were compared to two control groups: one that received a sham injection of corn oil, and 
a group that did not receive any injection. When chicks' behavior was compared across experimental conditions, the mean duration in tonic immobility was significantly higher in testosterone treated chicks when compared to sham and control chicks. Testosterone treated chicks also displayed an increased postnatal growth rate over the course of early development (Bertin et al., 2009).

A related study found that Japanese Quail chicks exposed to pre-incubation elevation of testosterone took longer to begin distress vocalizations and produced less total distress calls in an open field when compared to control chicks (Daisley, Bromundt, Mostl, \& Kotrschal, 2004). It also took longer for the testosterone-treated Japanese Quail to display tonic immobility. The results from these studies suggest that quail treated prenatally with elevated levels of steroid androgens display modified behavioral as well as physiological outcomes following hatching.

\section{Elevated yolk androgens affect prenatal and postnatal perception}

It has long been recognized that many precocial avian species show responsiveness to auditory stimulation several days before hatching (Gottlieb, 1968), and as such a form of prenatal auditory learning is possible. It is also known that augmented prenatal sensory experience can interfere with auditory learning in birds (Radell \& Gottlieb, 1992). In addition to modification of sensory experience, hormones of maternal origin have also been found to modify prenatal auditory learning in birds.

Bertin et al. (2009) presented evidence that exposure to elevated prenatal testosterone enhances prenatal auditory learning in bobwhite quail chicks when compared to control chicks. Twenty four hours prior to hatch, quail embryos were exposed to an individual maternal assembly call (Call A or Call B) and then tested twenty four hours 
following hatch. The eggs that had received elevated yolk testosterone prior to incubation were found to learn the prenatal call with less exposure time than control groups that did not receive elevated levels of testosterone. To date, very little is known about the effects of prenatal androgen levels on postnatal auditory perceptual learning. Gestagens in the pre-incubated egg

Gestagens are found in large amounts in the pre-incubated eggs of birds (Bertin et al., 2009; Gil, 2003; Groothuis \& Schwabl, 2008). Gestagens are a class of hormone that includes progesterone, which acts as a precursor to the production of both androgens and glucocorticoids over the course of avian prenatal development (Henrikson et al., 2011). Interestingly, Henriksen, Groothuis, and Rettenbacher (2011) recently found that female chickens (Gallus gallus domesticus) with elevated plasma corticosterone levels deposit fewer gestagens and androgens into their egg yolks, which suggests an inverse relationship between maternal stress and progesterone content in the yolk of their eggs. A study with Japanese Quail tested steroid yolk concentrations in birds that had been artificially selected for levels of inherent fearfulness (Bertin, Richard-Yris, Houdelier, Richard, Lumineau, Kotrschal, \& Mostl, 2009). A line of birds that were more susceptible to remain in tonic immobility were bred to form a divergent line of quail that displayed high levels of tonic immobility (Bertin et al., 2009). In laying hens of the more fearful quail line, progesterone and testosterone were found to be less concentrated in the egg yolk of pre-incubated eggs. Hens that were more fearful produced chicks that were more likely to be fearful themselves, but only because they were bred with males who were from the same divergent line. Postnatal auditory learning was not tested in the Japanese Quail study, so a direct comparison to the current 
study cannot be made, but the authors findings did suggest a strong relationship between progesterone concentrations in pre-incubated egg yolk and offspring phenotype.

These results suggest that even if no difference exists in the concentration of corticosterone in the pre-incubated egg of stressed and un-stressed females, the embryo's corticosterone exposure may still differ as a result of differential gonadal steroid concentration in the yolk. The aforementioned effect may also shed light on why the effects of maternal plasma glucocorticoids in avian species affect offspring phenotype, even when high levels of corticosterone are not transferred to the egg yolk.

\section{Summary}

The present dissertation project is a continuation of my master's thesis project, in which yolk from bobwhite quail eggs were experimentally manipulated to elevate levels of progesterone, a gestagen steroid of maternal origin. Progesterone is the most abundant of all androgens found in the pre-incubated yolk of Northern bobwhite quail (Bertin et al., 2009). Variation of progesterone is found between eggs and is related to egg weight (Bertin et al., 2009).

In my previous study, pre-incubated eggs were injected with corn oil that contained enough dissolved progesterone to elevate yolk levels two standard deviations above normal levels found in pre-incubated bobwhite quail eggs. The experimental elevation of progesterone was made by infusing $826 \mathrm{ng}$ of steroid progesterone into $20 \mu \mathrm{l}$ of corn oil. Two additional experimental control groups were formed by injecting eggs with either the oil vehicle only, or with no injections. Compared to the two control groups, the eggs that were treated with progesterone prior to incubation produced chicks 
that were more emotionally reactive when exposed to several standard tests of fear and stress related behavior (Herrington \& Lickliter, 2012).

The present dissertation project demonstrates the usefulness of studies using precocial birds to examine the prenatal effects of elevated hormones of maternal origin and their effects on postnatal behavior. For numerous reasons already mentioned, studies with mammalian subjects are difficult, as there are many more prenatal and early postnatal experiences that can influence behavior and physiology. 


\section{CHAPTER IV}

\section{RESEARCH DESIGN}

The present dissertation was designed to expand on the findings from my master's thesis study (Herrington, 2012). My master's thesis study was the first to examine the effects of elevated prenatal progesterone on neonatal emotional reactivity in bobwhite quail. In my master's thesis study, I experimentally elevated naturally occurring levels of progesterone via injections of progesterone infused corn oil into the yolks of bobwhite quail eggs. Weekly batches of progesterone treated eggs were incubated and reared with two experimental control groups: a corn-oil vehicle injected control group and a nonmanipulated control group. Individual chicks from the $\mathrm{P}, \mathrm{V}$, and $\mathrm{C}$ groups were given three classic tests of emotional reactivity: a tonic immobility task, a hole-in-the-wall emergence task, and an open field task. Results from the tonic immobility task and the hole-in-the-wall emergence task suggested that the P chicks had enhanced emotional reactivity compared to the two control groups.

Although the results from Herrington (2012) suggested that elevated levels of yolk progesterone can affect chicks' emotional reactivity, it was unclear if progesterone modifies other behaviors such as learning and memory. In addition, it was unknown if the elevation of prenatal progesterone prior to incubation resulted in higher levels of progesterone in the egg yolk across prenatal development. As previously discussed, progesterone is a precursor to glucocorticoid production in avian eggs (Groothuis et al., 2008), and the current study was designed in part to test whether elevation of progesterone prior to incubation can indirectly elevate yolk corticosterone levels across the prenatal period. The present dissertation study was also designed to test the effects of 
elevated prenatal progesterone levels on embryonic growth and development. As previously mentioned, the presence of high levels of corticosterone in egg yolk has been shown to affect postnatal growth and development in precocial birds (Hayward et al., 2006).

Experiment 1 was designed to examine the effect of elevated progesterone on yolk hormone concentrations across prenatal development. To date, no study has explored the effects of elevated prenatal progesterone on embryonic growth and development in bobwhite quail. Samples from individual egg yolks were collected from eggs that were treated with progesterone, eggs that received a corn-oil vehicle injection only, and non-manipulated eggs. High Performance Liquid Chromatography with tandem Mass Spectroscopy (HPLC/MS) verified the presence and concentration of progesterone in individual egg yolks. In addition, physiological measures such as embryonic weight and embryonic length were recorded across prenatal development to determine the effects of prenatal progesterone elevation on embryonic growth rate.

In Experiment 2, the effect of elevated prenatal progesterone on prenatal auditory learning was explored. Previous research has found that that artificial elevation of testosterone prior to incubation results in augmented prenatal learning and memory when compared to chicks hatched from eggs with no testosterone elevation (Bertin et al., 2009). To examine the effects of elevated prenatal progesterone on prenatal auditory learning, bobwhite quail embryos that had received 1) elevation of progesterone prior to incubation, 2) an injection of corn-oil, and 3) no injection were exposed to an individual bobwhite maternal call 24 hours prior to hatching. Chicks were then tested at 24 and 48 hrs after hatching to determine if they remembered the familiarized maternal call. 
Experiment 3 was designed to compare postnatal auditory learning in chicks hatched from progesterone-treated eggs to chicks that had not received prenatal elevation of progesterone. Chicks exposed to elevated levels of progesterone prior to incubation were reared with chicks that had not received prenatal progesterone elevation, and were passively exposed to an individual maternal assembly call (Call A or Call B) for a period of 24 hours immediately following hatching. Individual chicks were then tested at 24 and 48 hours after hatch for their preference for the familiarized maternal call over a novel variant of the bobwhite maternal call.

The following research questions were examined (predicted results are shown in Tables 2 and 3):

Experiment 1: After elevation of progesterone prior to incubation, will the steroid content of the eggs differ from eggs that have not been treated with progesterone across prenatal development?

Hypothesis 1: Artificial elevation of progesterone before incubation should elevate the levels of progesterone found in the egg yolk of treated bobwhite quail eggs compared to bobwhite quail eggs that had not been treated with progesterone. It is predicted that the concentration of progesterone in bobwhite quail egg yolks will be significantly higher across prenatal development in eggs treated with progesterone compared to eggs that were not treated with progesterone. 
Experiment 2: When chicks are simultaneously presented with a novel bobwhite maternal call and a bobwhite maternal call that they were exposed to prenatally ( 24 hours prior to hatching), will progesterone-treated birds show a preference for the familiarized call?

Hypothesis 2: Bobwhite quail chicks that hatch from eggs with experimentally elevated progesterone in the pre-incubated yolk should form an enhanced postnatal preference for a species-specific maternal call (Call A and Call B) after prenatal exposure to the call 24 hours prior to hatch. It is predicted that control groups (oil vehicle and no injection) will show a postnatal preference for the familiarized maternal call, based on previous findings from quail chicks with elevated yolk androgens (Bertin et al., 2009).

Table 2

Hypotheses for Experiment 2

\begin{tabular}{|c|c|c|c|}
\hline Group & $\begin{array}{c}24 \mathrm{Hr} \\
\text { Condition }\end{array}$ & $\begin{array}{c}\mathbf{4 8} \mathrm{Hr} \\
\text { Condition }\end{array}$ & Preference \\
\hline Progesterone & $\mathrm{N}=30$ & $\mathrm{~N}=30$ & $\mathrm{EP}$ \\
\hline Control & $\mathrm{N}=30$ & $\mathrm{~N}=30$ & $\mathrm{P}$ \\
\hline Oil & $\mathrm{N}=30$ & $\mathrm{~N}=30$ & $\mathrm{P}$ \\
\hline
\end{tabular}

Predicted preferences at 24 and $48 \mathrm{hrs}$ after hatch in each experimental group for a familiarized call presented $24 \mathrm{hrs}$ post hatch

Experiment 3: Will birds that hatch from progesterone-treated eggs form a preference for an individual maternal call that was presented to them postnatally (24 hours after hatch)? 
Hypothesis 3: Elevation of prenatal progesterone levels will enhance postnatal learning of the familiar species-specific bobwhite maternal assembly call. It is expected that chicks from the two control groups will form a preference for a familiarized bobwhite maternal assembly call after 24 hours of passive exposure immediately following hatching. In contrast, I predict the progesterone-treated eggs will produce chicks that will show an enhanced preference for the familiarized maternal call after 24 hours of passive exposure immediately following hatching.

\section{Table 3}

Hypotheses for Experiment 3

\begin{tabular}{|c|c|c|c|}
\hline Group & $\begin{array}{c}24 \mathrm{Hr} \\
\text { Condition }\end{array}$ & $\begin{array}{c}48 \mathrm{Hr} \\
\text { Condition }\end{array}$ & Preference \\
\hline Progesterone & $\mathrm{N}=30$ & $\mathrm{~N}=30$ & $\mathrm{EP}$ \\
\hline Control & $\mathrm{N}=30$ & $\mathrm{~N}=30$ & $\mathrm{P}$ \\
\hline Oil & $\mathrm{N}=30$ & $\mathrm{~N}=30$ & $\mathrm{P}$ \\
\hline
\end{tabular}

Predicted preferences in each experimental group when tested at 24 and $48 \mathrm{hrs}$ after hatch for a familiarized call presented for 24 hours post-hatch 


\section{CHAPTER V}

\section{GENERAL METHOD}

\section{Subjects}

Subjects were 270 incubator-reared bobwhite quail chicks (Colinus virginianus). Fertilized, unincubated eggs were received weekly from a commercial supplier (Strickland, GA) and set in an incubator maintained at approximately $75-80 \%$ relative humidity and $37.5^{\circ} \mathrm{C}$. Embryonic development was tracked from incubation day 0 until hatch. To control for possible variations in developmental age, only chicks that hatched on day 23 of incubation were used in these series of experiments. Following hatching, groups consisting of 8-12 subjects were housed in a clear plastic rearing tub until testing. Chicks had access to food and water ad libitum except during testing. Ambient air temperature in both the rearing room and testing room was maintained at approximately $30^{\circ} \mathrm{C}$. The total number of subjects are listed in Table 4 below.

Table 4

Total Number of Subjects Tested in All Experiments

\begin{tabular}{|c|c|c|c|}
\hline Group & $\begin{array}{c}\text { Experiment } \\
\mathbf{1}\end{array}$ & $\begin{array}{c}\text { Experiment } \\
\mathbf{2}\end{array}$ & $\begin{array}{c}\text { Experiment } \\
\mathbf{3}\end{array}$ \\
\hline Progesterone & $\mathrm{N}=30(1)$ & $\mathrm{N}=60(3)$ & $\mathrm{N}=60(2)$ \\
\hline Oil & $\mathrm{N}=30$ & $\mathrm{~N}=60(2)$ & $\mathrm{N}=60(3)$ \\
\hline Control & $\mathrm{N}=30$ & $\mathrm{~N}=60(5)$ & $\mathrm{N}=60(5)$ \\
\hline
\end{tabular}

* Numbers in parenthesis are subjects that were not included in the analysis. Subjects removed from Experiments 2 and 3 were non-responders during behavioral testing (did not move from starting position). Subject removed from Experiment 1 (Prenatal Day 7) was an outlier (concentration was more than twice the standard deviation from the mean). 


\section{Progesterone elevation procedure}

Fertile bobwhite quail eggs were separated into 3 conditions for use in all 3

Experiments. A total of 30 eggs were used from each experimental group (C, V, and $\mathrm{P})$. Eggs were selected from a shipment of 200 eggs for this experiment every week starting in the month of April until a total of 30 eggs per group were injected. The experimental condition consisted of progesterone-treated eggs that were injected prior to incubation with approximately $826 \mathrm{ng}$ of progesterone infused in $20 \mu \mathrm{l}$ corn oil vehicle (P), which elevated the level of progesterone found in the egg 2 standard deviations above normal levels previously reported in this species (Bertin et al., 2009). The two control groups consisted of eggs injected with a corn oil vehicle only $(\mathrm{O})$ and eggs that received no injection (C). All eggs were weighed and matched across conditions. To stay within the physiological range of known levels of yolk progesterone, only those eggs that weighed between $9.18 \mathrm{~g}$ and $10.86 \mathrm{~g}$ were used. To deliver the corn oil and progesterone to the yolk, the area above the air sack of the egg was sanitized by swabbing the area with $70 \%$ isopropyl rubbing alcohol, and a small hole was made with a sterile 26 gauge needle. An additional sterile 26 gauge needle and glass syringe delivered the progesterone infused corn oil and corn oil only into the egg yolk. Once the corn oil and progesterone infused corn oil had been delivered to the yolk, the hole was covered with a small piece of sterile egg shell and surgical glue.

Analysis of progesterone in egg yolk/embryonic growth

Prior to HPLC/MS analysis, eggs from the $\mathrm{P}, \mathrm{V}$, and $\mathrm{C}$ groups were placed in a 20C freezer until analysis. After the eggs were frozen, the shell and albumen of the eggs was removed and a lateral cross section of the yolk was made with a scalpel. We did this 
because in other bird species, progesterone is most concentrated in the outer layer of the yolk (Bertin et al., 2009; Hackl, Bromundt, Daisley, Kotrschal, K., \& Mostl, E., 2003). Samples were then homogenized with an electric mixer for 10 minutes, and the steroids were extracted according to a protocol for extraction in a previous study in quail (Hackl et al., 2003).

Each $.5 \mathrm{~g}$ sample of yolk was suspended in $8 \mathrm{ml}$ of methyl alcohol and $1.5 \mathrm{ml}$ of milli-Q water, then vortexed for $1 \mathrm{~min}$. A $10 \mathrm{ml}$ sample of the supernatant was extracted from the individual samples after 10 minutes of centrifugation at $-10^{\circ} \mathrm{C}$ and $4000 \mathrm{rpm}$. Hormones were extracted in ethanol from the yolk supernatant by solid phase extraction (SPE) with 10ml C18 Sep Pak cartridges (Sigma) and a vacuum extractor apparatus. The C18 Sep Pak cartridges were conditioned with methanol, and then with milli-q water. We then passed the samples through the conditioned C18 Sep Pak cartridges, and washed the cartridges with milli-q water after all of the sample had been passed through. We then eluted the samples with $99 \%$ ethanol for analysis, and evaporated the samplescalib in a vacuum tube. Samples were re-suspended in $200 \mu 1$ of acetonitrile HPLC/MS assay buffer and immediately analyzed or the samples were stored in acetonitrile assay buffer at $-20^{\circ} \mathrm{C}$ until analysis.

Relative peaks for progesterone were obtained via High Performance Liquid Chromatography with Tandem Mass Spectroscopy (HPLC/MS). Use of HPLC/MS resulted in a high level of experimental sensitivity, and ensured that cross-reactivity with similar hormones was minimized. It is known that steroid androgens tend to cross-react in more widely used methods such as ELISA, EIA, and RIA antibody assays (see Rettenbacher et al. 2005 for review). This was the first study that employed HPLC/MS 
to determine steroid hormone content in the egg yolk of Northern bobwhite quail. The method I used for the progesterone analysis was based on a previous HPLC/MS method to measure progesterone in chicken egg yolk (Yang, Shao, Zhang, Wu, \& Ying, 2008) with some changes. The HPLC portion was the same with the exception that the flow rate was $0.5 \mathrm{ml} / \mathrm{min}$ instead of $0.3 \mathrm{~mL} / \mathrm{min}$. For the MS portion, analysis was done in full scan mode (scan range m/z 200-1000) and multiple reaction monitoring (MRM) mode with three parent-product ion transitions (315 -> 279, 315 -> 297, and 315 -> 97). The collision energy for the first two was $15 \mathrm{~V}$ and for the last one was 35V. Quantitation was done with the transition $315->97$.

To determine if elevated progesterone affected prenatal growth and development of the fetus, a series of measurements were on odd prenatal developmental days 0-17. While the egg yolk samples were being taken on odd days of development $(\mathrm{N}=3$ from the $\mathrm{P}, \mathrm{V}$ and $\mathrm{C}$ groups on odd prenatal days $0-17$ ) the physical growth of the embryo was also recorded. Leg length, body length, and skull length as well as chick weight and total yolk weight were recorded from incubation through odd prenatal days 0-17.

\section{Behavioral testing apparatus}

In the prenatal learning experiment, on day 22 of incubation approximately 24 hours prior to hatch, the eggs were transferred to a sound-attenuated room and placed in a portable incubator. The incubator was maintained at $30^{\circ} \mathrm{C}$ with a relative humidity of approximately 65\%. For Experiments 1 and 2, a speaker delivered a species-specific maternal assembly call (either Call A or Call B) 10-mins per hour for a total of 24-hrs (total exposure of 240 minutes), broadcast by way of a customized computer program. We counterbalanced the exposure of Call A with Call B (individual maternal assembly 
calls) at the time of exposure and testing. For the postnatal learning experiment, at approximately 24 hours after hatch, chicks were transferred to a sound-attenuated room into a clear rearing tub. A speaker was placed outside of the tub and the maternal call (Call A or Call B) was played from the speaker by means of a custom computer program.

Tests of chick behavior were conducted in a circular arena $130 \mathrm{~cm}$ in diameter, and $60 \mathrm{~cm}$ in height. The surface of the arena is painted white, and an opaque white curtain shrouds the wall of the arena. Chicks were released into the open field arena, and behavior was observed for a total of $300 \mathrm{sec}$ per subject per trial in Experiments 2 and 3. A color digital video camera is mounted directly above the arena to record behaviors and allow for automated behavioral testing. A total of $5 \%$ of the arena was demarcated to two semi-circular approach areas (see figure 2). The arena and approach areas were denoted by Noldus Ethovision XT 8.5 software (Wageningen, The Netherlands). While the bird was in the testing arena, two opposing speakers played either the familiar maternal call or a novel variant of the maternal call from the two approach areas. The decibel levels produced by speakers at prenatal training, postnatal training, and testing were calibrated to 65 decibels using a Sinometer digital sound level meter (Union City, CA). Ambient room temperature was held at a constant for testing all subjects $\left(91^{\circ} \mathrm{F}\right)$, as was the light source (a florescent light above the arena). Two portable heaters were located outside of the arena behind the speakers that were projecting the auditory stimulus. Off-white blankets were draped on the walls in the room where the arena was located so that the chicks would not be attracted to visual irregularities. 


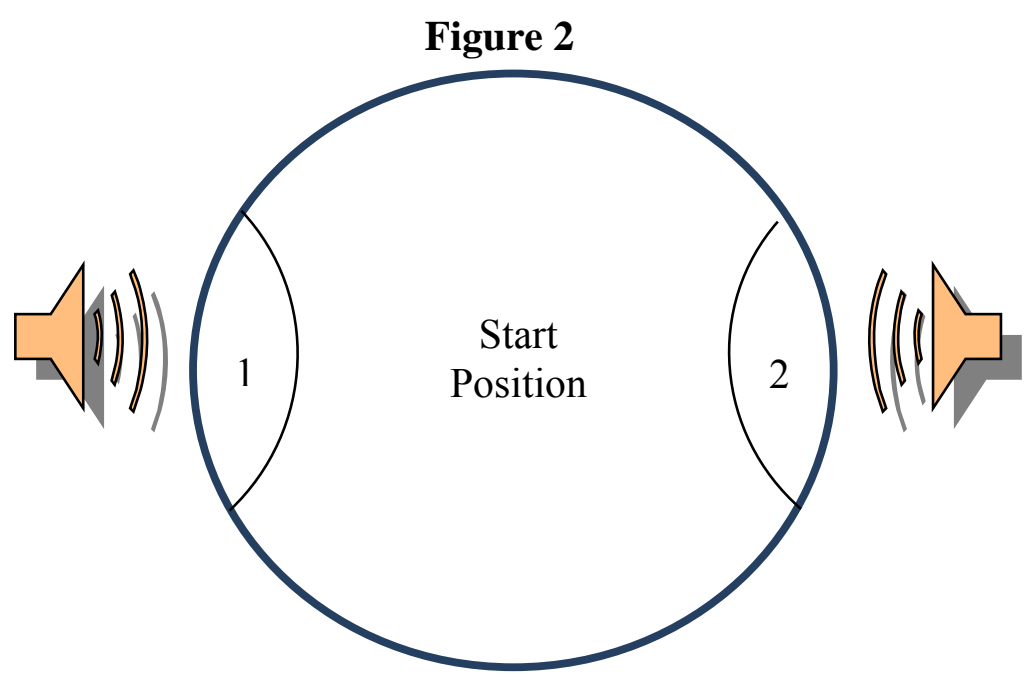

Figure 2. The Behavioral Testing Area. Area one (1) and area two (2) represent a total of 5\% of the entire arena space. Speakers imbedded in the wall of the arena played variants of the maternal call. Call location was counterbalanced across testing times.

Method for experiment 2: prenatal auditory learning

One hundred and eighty bobwhite quail chicks were divided into three conditions: progesterone-treated $(\mathrm{P})$, oil-vehicle injected $(\mathrm{V})$, and non-manipulated eggs $(\mathrm{C})$. Embryos in the $\mathrm{P}, \mathrm{V}$, and $\mathrm{C}$ conditions received prenatal auditory stimulus exposure using a protocol outlined in previous studies of bobwhite quail embryos (e.g., Honeycutt \& Lickliter, 2001, 2002; Lickliter et al., 2002; Reynolds \& Lickliter, 2004). On the morning of the $21^{\text {st }}$ day of incubation, embryos were transferred to a portable Reptibator hatcher and maintained at $37.5^{\circ} \mathrm{C}$ and approximately $60 \%$ humidity. In the morning of Day 22 of incubation, a stimulation procedure began. The auditory stimulus for prenatal stimulation was a recording of an individual bobwhite quail maternal call. A loop of the 
call was broadcast at a rate of 13 calls per minute. The call was broadcast for 10 minutes every hour during the 24 hour period prior to hatch, which resulted in a total of 240 minutes of total exposure. The sound level of the bobwhite quail maternal call was kept at a constant $65 \mathrm{~dB}$.

Preference for the familiarized bobwhite maternal call vs. a novel bobwhite maternal call was tested in $\mathrm{P}, \mathrm{V}$, and $\mathrm{C}$ chicks at $24(\mathrm{~N}=90)$ and $48(\mathrm{~N}=90)$ hrs after hatching. Different chicks were tested at 24 and $48 \mathrm{hrs}$ after hatching in order to control for habituation to the learned bobwhite maternal call. Individual chicks were carried to a sound-attenuated testing arena in a wooden box $(10 \mathrm{~cm} \times 10 \mathrm{~cm} \times 10 \mathrm{~cm})$. All tests of preference consisted of a 5-min simultaneous choice test between the two bobwhite quail maternal assembly calls (familiar v unfamiliar). The arena where testing took place was circular $($ height $=24 \mathrm{~cm}$; diameter $=130 \mathrm{~cm})$ and painted white with white curtains covering the walls. A digital video camera located above the arena recorded the location of the chick in the circular arena. Noldus Ethovision XT 8.5 software automatically tracked the position of the chick in the arena. Two speakers were located on opposite sides of the testing arena, and simultaneously broadcasted two variations of a bobwhite quail maternal assembly call (Call A and Call B). The calls were counter-balanced between the two sides of the arena. Both Call A and Call B are similar in phrasing, frequency modulation, and repetition rate, and vary in minor peaks of frequency and pitch (Harshaw \& Lickliter, 2007). Chicks were tested between 12pm and $6 \mathrm{pm}$, and only if they were awake during time of testing. Previous studies have shown that bobwhite quail neonates do not show a naïve preference for these two calls (Honeycutt \& Lickliter, 2001, 2002). Two semicircular approach areas were created that encompass 
the speaker area where Call A and Call B was broadcast, which corresponds to $5 \%$ of the total surface area of the arena. Entrance into the approach areas was monitored by Ethovision XT 8.5 automated behavioral observation software.

Method for experiment 3: postnatal auditory learning

One hundred and eighty eggs from the $\mathrm{P}, \mathrm{V}$, and $\mathrm{C}$ conditions were incubated and hatched according to the protocol described in Experiment 2. Immediately after hatching, bobwhite quail neonates from all three experimental conditions $(\mathrm{P}, \mathrm{V}$, and $\mathrm{C})$ were transferred to a clear rearing tub in a sound attenuated stimulation room. Before transfer, chicks were labeled by a colored leg band to denote their experimental condition. Chicks from each experimental group were reared together in the same tub to ensure homogeneity in the postnatal rearing environment.

A speaker was placed on the outside of the rearing tub, which played an individual bobwhite maternal assembly call (Call A or Call B). Chicks from all three conditions were exposed to the maternal call for a period of 10 minutes every hour for 24 hours, resulting in a total exposure of 240 minutes for either Call A or Call B. After 24 hours of exposure to the auditory stimulus, the rearing tubs were transferred to a rearing room for 24 hours. At 24 and 48 hours post-hatch, birds were individually tested for their preference for the familiarized maternal call vs. the novel call. Testing protocol for assessing auditory preference followed the same procedure outlined in Experiment 2. Data analysis

In the prenatal and postnatal learning experiments, determination of preference for the maternal calls presented during testing was determined by the duration of time spent in the approach area of the familiar or novel call. A preference was inferred for 
either the familiar or novel maternal call (Call A or Call B) based on 1) the raw duration time (in seconds) spent in the familiarized call zone and 2) the proportion of total duration score. The proportion of total duration time (PTDT) scores are derived by taking the time spent in the 'familiar' approach area over the total time spent in both approach areas. Chicks that do not spend at least 30 seconds in an approach area were scored as a non-responder and were not included in the analysis. The total non-responder rate for each group was as follows: Chicks were scored as showing a preference for the familiarized or novel maternal call if the chick spends at least 30 seconds in an approach area and at least twice as long in that approach area as the other.

The data was not normally distributed for the raw-duration scores in the familiar and novel approach zones and normally distributed for the PTDT scores. Appropriate omnibus and post-hoc tests in SPSS were used to assess differences between the experimental conditions. The variability of yolk hormone levels were analyzed by way of a univariate regression model using SPSS software. The model tracked changes over time to levels of progesterone in the bobwhite quail egg yolks across prenatal days 0-17. One outlier was removed from the progesterone-treated group at developmental day 13 (the detected levels of progesterone were two times the standard deviation of the sample mean). 


\section{CHAPTER VI}

\section{EXPERIMENT 1}

\section{Hormone content and growth in prenatal development}

It is unclear at this point how elevation of progesterone prior to incubation affects the hormone content of the egg yolk across prenatal development. The primary purpose of Experiment 1 was to track the levels of progesterone in the yolk across prenatal days 0-17 in the $\mathrm{P}, \mathrm{V}$, and $\mathrm{C}$ conditions. The prediction was that the presence of elevated progesterone levels in pre-incubated eggs would result in higher levels of progesterone in the egg across prenatal development.

A previous study found no significant differences in postnatal chick weight and growth rate in eggs treated prenatally with progesterone compared to chicks that hatched from oil vehicle injected and non-injected eggs (Herrington, 2012). The effect of prenatal progesterone elevation on bobwhite quail embryonic growth is unknown. A secondary analysis of embryonic growth was conducted to determine if prenatal progesterone elevation affected embryonic growth.

\section{Results}

There was a significant main effect, $\mathrm{F}(2,87)=3.282, p=.044$, found between the P group and both control groups that indicated progesterone levels were higher across prenatal development in the P group (see Figure 4). In addition, there was a significant main effect of day that showed progesterone levels decrease over time in all three experimental groups, $\mathrm{F}(4,87)=20.83, p=.001$. When developmental days were collapsed into early, middle, and late development, univariate regression found that detectable levels of progesterone in the yolk were significantly higher than the two 
control groups $(\mathrm{p}<.001$; see Figure 5$)$. No significant differences in progesterone levels were found between the $\mathrm{C}$ and $\mathrm{V}$ control groups $(p>.05)$. Multiple tests were run to determine any main and interaction effects between groups across days 1 through 17 of prenatal development. No significant interaction effects were found in a comparison between condition and developmental day, $\mathrm{F}(8,87)=.317, p>.05$.

There were no significant differences in physiological development found between the experimental groups from prenatal day 0 through prenatal day $17(p>.05$ in all ANOVA and post-hoc Tukey tests).

\section{Figure 3}

HPLC/MS Relative Peaks for Progesterone

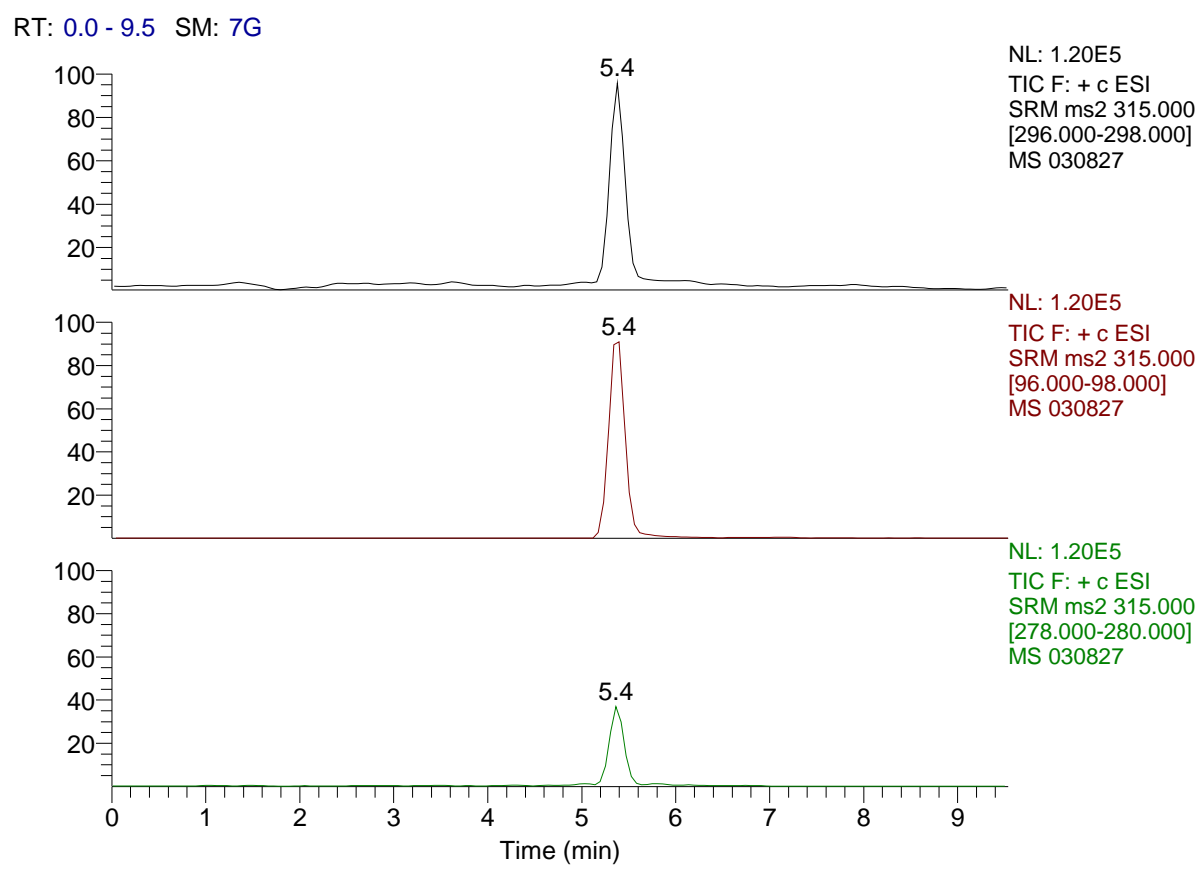

Fig 3. This figure displays relative peaks for three separate fractions of progesterone following HPLC/MS analysis. These peaks occur at the same time for each of the different fractions of progesterone, which strongly suggests that progesterone is the only substance that is being quantified by the HPLC/MS analysis. 


\section{Figure 4}

Average change of Progesterone Levels Across Prenatal Development

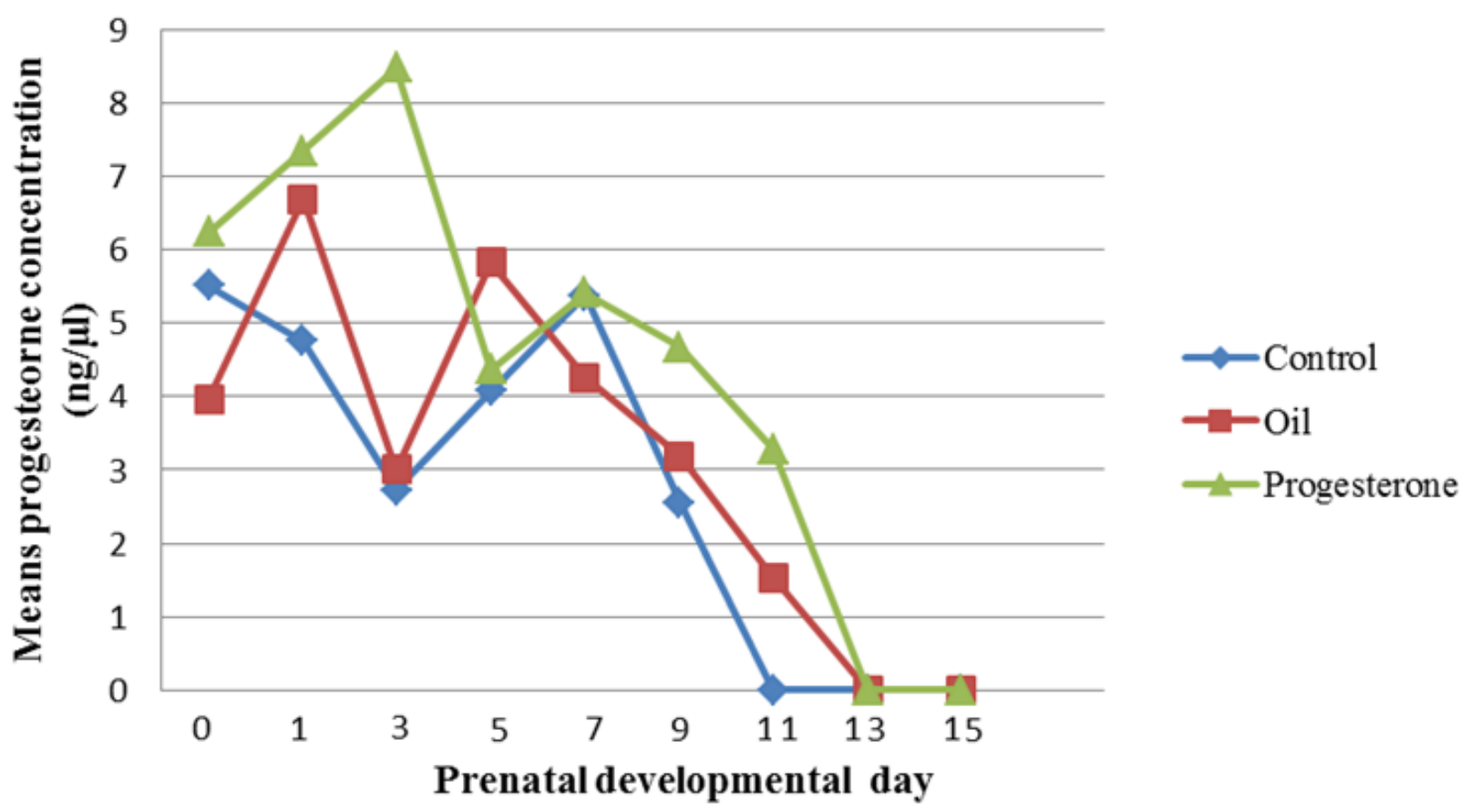

Fig 4: A graphical representation of the mean levels of progesterone found in bobwhite quail eggs across individual days of prenatal development (days 1-17). There was a significant main effect ( $p=.044$ ) of higher progesterone levels over developmental time in $\mathrm{P}$ eggs compared to $\mathrm{V}$ and $\mathrm{C}$ eggs. A second main effect showed that progesterone levels decreased significantly over time in all experimental groups $(p=.001)$. 


\section{CHAPTER VII}

\section{EXPERIMENT 2}

\section{Effects of prenatal progesterone elevation on prenatal auditory learning}

A previous study found that bobwhite quail chicks hatched from eggs that had artificially elevated levels of progesterone prior to incubation are more emotionally reactive compared to chicks hatched from eggs that did not receive progesterone prior to incubation (Herrington, 2012). It remains unclear what influence artificially elevated prenatal progesterone prior to incubation has on prenatal auditory learning in bobwhite quail. Experiment 2 sought to determine if elevation of progesterone prior to incubation affects prenatal auditory learning in bobwhite quail embryos. To test for any age-related effects on prenatal auditory learning, individual chicks from progesterone-treated (P), oil vehicle (V), and control groups (C) were tested at 24 and $48 \mathrm{hrs}$ after hatching. Results for the 24-hr condition

ANOVA and post-hoc Tukey tests were performed on the proportion of total duration time (PTDT) spent in the approach area with the prenatally familiarized version of the call against the chance value (.50) for the P, V, and C groups. The mean PTDT scores indicated that the P-treated group had a lower mean of duration time spent in proximity to the familiar call $(M=.40, S D=.27)$ than the $\mathrm{V}(M=.70, S D=.22)$ and $\mathrm{C}(M$ $=.68, S D=.22$ ) when tested at 24 hours of age. A One-Way ANOVA revealed the between-group differences were significant, F $(2,87)=15.07, p=.001$. Post-hoc Tukey's HSD tests indicated the mean PTDT score to the familiar call in the P group was significantly less than the PTDT scores of the $\mathrm{V}$ and $\mathrm{C}$ groups $(p>.05)$. The time spent in the familiar approach zone for the V and $\mathrm{C}$ group did not differ, as shown in Figure 6 
below $(p>.05)$. One sample t-tests were run on each experimental condition against a value of .5 to determine if the chicks showed a significant preference for the familiar or the novel maternal bobwhite assembly call. The $\mathrm{C}$ group showed a significant preference for the familiar call, $t(29)=4.61, p=.001$ as did the V group, $t(29)=4.97, p=.001$, while a preference for the novel call approached significance in the P group, $t(29)=-.202$, $p=.053$.

\section{Figure 6}

Mean PTDT for Familiar and Novel Call in the Prenatal 24-hr Condition

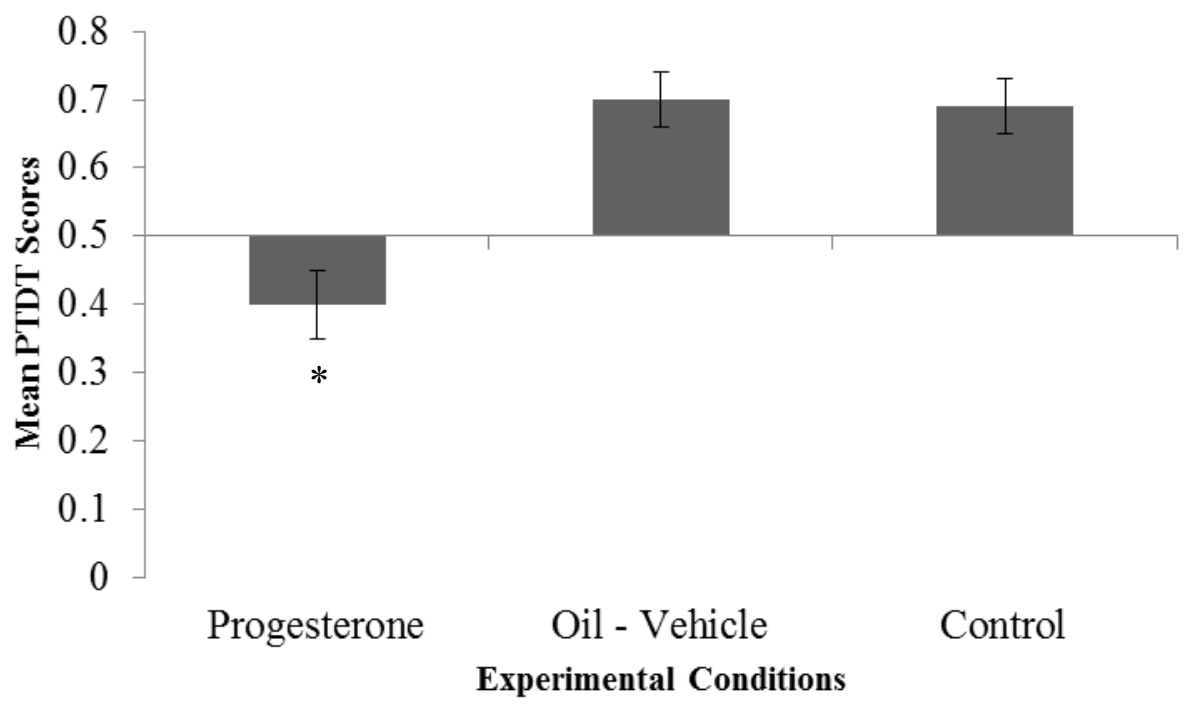

Fig 6. Mean \pm SEM percentage of total duration time scores. Values above .5 indicate a preference for the familiar call, while values below .5 indicate a preference for the novel call. Significance indicated by post-hoc Tukey's HSD. ${ }^{*} p<0.05$.

Results for the 48-hr condition

Data from the 48-hr condition was analyzed with a one-way ANOVA and post-hoc Tukey's HSD tests. The analysis was performed on the proportion of total duration time (PTDT) spent in the approach area with the prenatally familiarized version of the call 
against the chance value (.50) for the $\mathrm{P}, \mathrm{V}$, and $\mathrm{C}$ groups. The mean PTDT scores (shown in Figure 7) indicated that the $\mathrm{P}$ treated group had a lower mean of duration time spent in proximity to the familiar call $(M=.42, S D=.34)$ than the $\mathrm{V}(M=.57, S D=.32)$ and $\mathrm{C}(M$ $=.55, S D=.24)$ at when tested $48 \mathrm{hr}$ after hatching. A One-Way ANOVA revealed the between-group differences were not significant, $\mathrm{F}(2,87)=2.012, p=.140)$. One sample t-tests were run on each experimental condition against a value of .5 to determine if the chicks showed a significant preference for the familiar or the novel maternal bobwhite assembly call. Results from the one sample t-tests indicated that no significant preference for the familiar or the novel call was shown by the experimental groups $(p>.05)$.

\section{Figure 7}

Mean PTDT for Familiar and Novel Call in the Prenatal 48-hr Condition

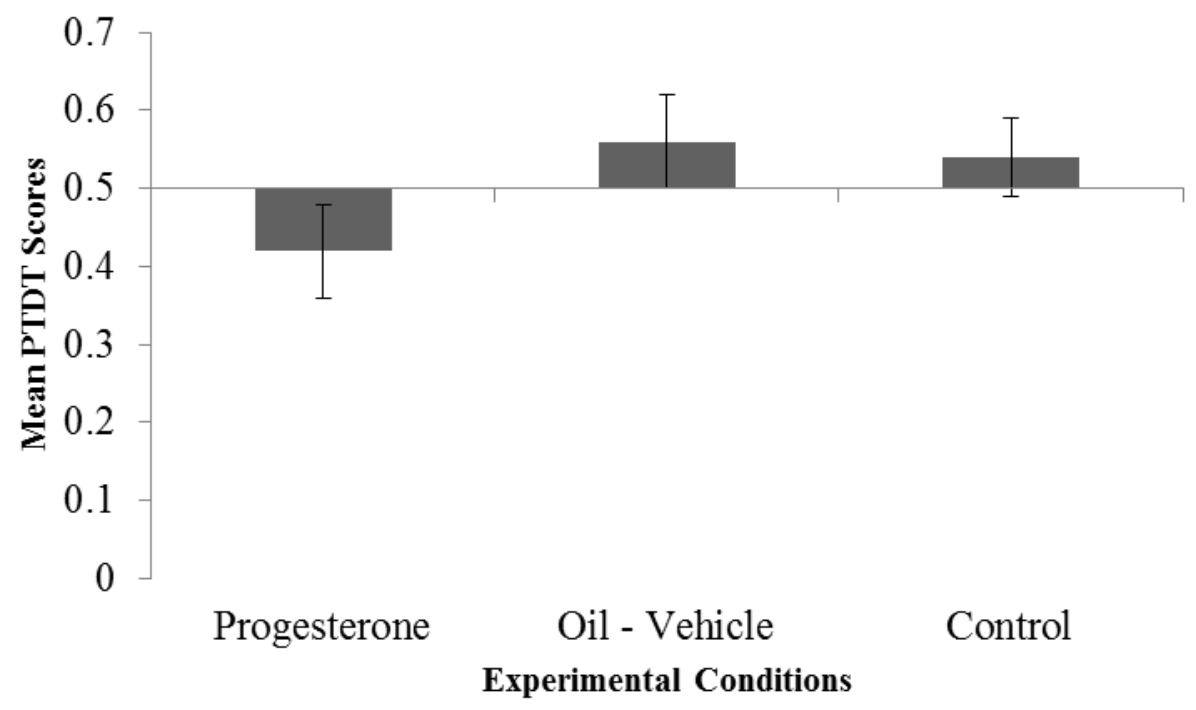

Fig 7. Mean \pm SEM percentage of total duration time scores. Values above .5 indicate a preference for the familiar call, while values below .5 indicate a preference for the novel call. Tukey's HSD between groups suggested no significant difference between groups ( $p>.05)$. 


\section{Summary}

The results from Experiment 2 in the $24 \mathrm{hr}$ condition agreed with the predictions made in the first hypothesis. Chicks that had received elevated levels of yolk progesterone prior to incubation did not show a preference for the familiarized bobwhite maternal call, while chicks in the $\mathrm{V}$ and $\mathrm{C}$ groups did show a significant preference for the familiarized bobwhite quail maternal call. Results from the 48-hr condition did not suggest a significant difference in time spent between groups in proximity to the familiar maternal bobwhite quail call. As shown in Figures 6 and 7, bobwhite quail chicks that had received elevated progesterone levels prior to incubation failed to exceed a chance score (.50), which suggested that they did not prefer the familiar bobwhite maternal call over the novel bobwhite maternal call. Both the $\mathrm{C}$ and $\mathrm{V}$ chicks showed a significant preference for the familiarized bobwhite maternal call in the 24-hr condition, while the $\mathrm{P}$ chicks did not; although they did form a preference for the novel call that approached significance. No significant preference for the novel or the familiar bobwhite maternal call was shown in the experimental groups in the 48-hr condition.

Overall, the results from Experiment 2 suggested that elevation of prenatal progesterone in egg yolk prior to incubation resulted in a diminished preference for the familiar maternal bobwhite assembly call. Will chicks that hatch from P-treated eggs display the same auditory learning deficit if they are exposed to a maternal call immediately after hatching? Experiment 3 was designed to address this question. 


\section{CHAPTER VIII}

\section{EXPERIMENT 3}

\section{Effects of prenatal progesterone elevation on postnatal auditory learning}

Experiment 3 was designed to test whether prenatal progesterone elevation affects postnatal auditory learning. Here, the design from experiment 2 was replicated, however the maternal call was presented to chicks immediately after hatching for a period of 24 hours (instead of 24 hours prior to hatching). It was hypothesized that chicks that hatch from progesterone-treated eggs will not form a preference for a bobwhite quail maternal call presented for 24 hours immediately after hatch when given simultaneous choice between the familiar bobwhite maternal call and a novel bobwhite maternal call.

Results for 24-hr condition

The Proportion of Total Duration Time (PTDT) scores met the assumption for parametric data (Levine's test $\mathrm{p}<.50$ ). A One-way ANOVA and post-hoc Tukey's HSD tests were performed on the PTDT spent in the approach area with the prenatally familiarized version of the maternal bobwhite quail call against the chance value (.50) for the $\mathrm{P}, \mathrm{V}$, and $\mathrm{C}$ groups. The mean PTDT scores indicated that the $\mathrm{P}$ group had a significantly higher mean of duration time spent in proximity to the familiar call $(M=.70$, $S D=.26)$ than the $\mathrm{V}$ group $(M=.52, S D=.21$ and $\mathrm{C}$ group $(M=.53, S D=.27)$ when tested at 24 hours after hatching. The One-way ANOVA revealed the between-group differences were significant, $\mathrm{F}(2,87)=5.396, p=.006$. The post-hoc Tukey HSD tests indicated the mean PTDT score to the familiar call in the P group was significantly greater than the PTDT scores of the $\mathrm{V}(p=.015)$ and $\mathrm{C}$ groups $(p=.016)$. One sample t-tests were run on each experimental condition against a value of .5 to determine if the chicks 
showed a significant preference for the familiar or the novel maternal bobwhite assembly call. The $\mathrm{P}$ group formed a significant preference for the familiar bobwhite maternal assembly call, $t(29)=4.34, p=.001$, while the $\mathrm{C}$ and $\mathrm{V}$ groups did not form a significant preference for the familiar bobwhite maternal assembly call ( $p=$ n.s. ). The mean PTDT scores for the $\mathrm{P}, \mathrm{V}$, and $\mathrm{C}$ groups are shown in Figure 8.

\section{Figure 8}

Mean PTDT for Familiar and Novel Call in the Postnatal 24-hr Condition

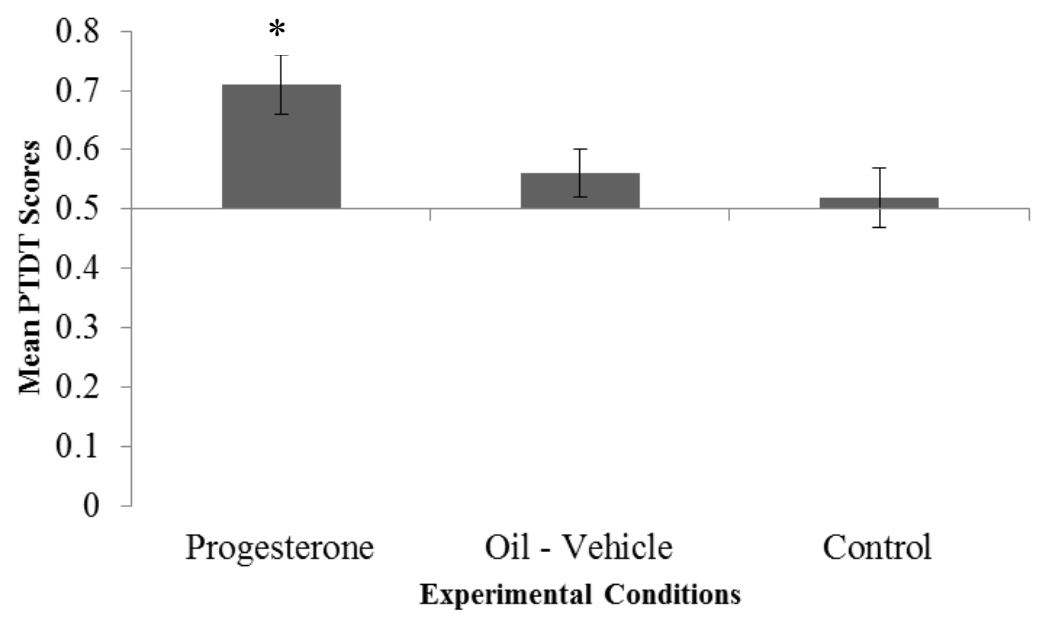

Fig 8. Mean \pm SEM percentage of total duration time scores. Values above .5 indicate a preference for the familiar call, while values below .5 indicate a preference for the novel call. Significance indicated by post-hoc Tukey's HSD. $* p<0.05$.

\section{Results for 48-hr condition}

Proportion of Total Duration Time (PTDT) scores met the assumption for parametric data with a Levine's test $(\mathrm{p}<.50)$. First, ANOVA and then post-hoc Tukey tests were performed on the PTDT spent in the approach area with the prenatally familiarized version of the call against the chance value (.50) for the $\mathrm{P}, \mathrm{V}$, and $\mathrm{C}$ groups. The mean PTDT scores indicated that the P-treated group had a significantly higher mean 
of duration time spent in proximity to the familiar call $(M=.79, S D=.23)$ than the $\mathrm{V}$ $(M=.60, S D=.24)$ and $\mathrm{C}(M=.58, S D=.23)$ when tested at 48 hours of age. A OneWay ANOVA revealed the between-group differences were significant, $F(2,87)=7.023$, $p=.001$. The post-hoc Tukey's HSD tests indicated the mean PTDT score to the familiar call in the $\mathrm{P}$ group was significantly greater than the PTDT scores of the $\mathrm{V}(p=.009)$ and $\mathrm{C}$ groups $(p=.003)$. Results from one sample t-tests showed that both the $\mathrm{V}$ group $t(29)=2.40, p=.023$, and the P group $t(29)=6.80, p=.001$ showed a significant preference for the familiar call, while preference for the familiar call approached significance in the C group, $t(29)=2.00, p=.060$.

\section{Figure 9}

Mean PTDT for Familiar and Novel Call in the Postnatal 24-hr Condition

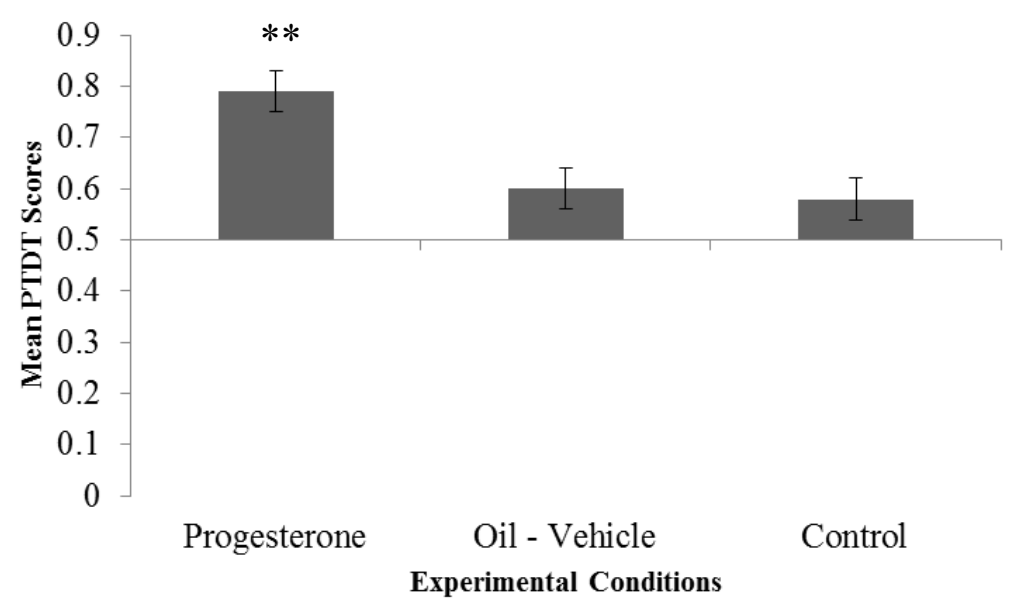

Fig 9. Mean \pm SEM percentage of total duration time scores. Values above .5 indicate a preference for the familiar call, while values below .5 indicate a preference for the novel call. Significance indicated by post-hoc Tukey’s HSD. ** $p<0.01$. 


\section{CHAPTER IX}

\section{GENERAL DISCUSSION}

The present study demonstrated that elevation of yolk progesterone via injections of progesterone-infused corn oil in pre-incubated bobwhite quail eggs alters prenatal and postnatal perceptual auditory learning in bobwhite quail chicks. Since Schwabl's discovery in 1993 that steroids of maternal origin are present in pre-incubated bird eggs, the majority of previous studies have injected maternally-derived hormones like corticosterone (Hayward, Richardson, Grogan, \& Wingfield, 2006) and testosterone (Bertin et al., 2009) into pre-incubated quail eggs to examine the effect of these hormones on prenatal and postnatal physiology and behavior. Since progesterone has never before been injected into quail eggs to study its effects on postnatal phenotype, the present study fills a specific gap in the literature.

Due to limitations within the current study, the mechanisms that underlie the effect of prenatal progesterone elevation on postnatal phenotype are still not well understood. Even so, comparisons of the findings in the current study to other studies that have experimentally manipulated hormone levels in bird eggs may shed some light on potential mechanisms by which yolk progesterone levels can influence postnatal phenotype. The following discussion section will 1) individually review the results from Experiments 1,2, and 3 in the current study and compare the results with previous findings in the literature, 2) propose potential mechanisms by which progesterone elevation could result in modification of prenatal and postnatal auditory perceptual learning, and 3) discuss limitations and directions for future study. 
Results from Experiment 1 suggested that prenatal progesterone concentrations were elevated across prenatal development in progesterone injected eggs compared to the two control conditions that did not receive progesterone treatment prior to incubation. In addition, progesterone was detected in greater quantities later in prenatal development (at prenatal day 13) in egg yolks that were injected with progesterone prior to incubation compared to control groups. No significant interaction effects were found between the experimental groups in Experiment 1, which is likely due in part to the low number of egg yolks that were analyzed ( $\mathrm{N}=3$ per experimental condition, per day).

In many species of bird, including bobwhite quail, progesterone is found in abundance in the egg yolk after egg formation and before incubation (Gil, 2003; 2008; Bertin et al., 2009). Progesterone is generally thought to load metabolic pathways for the production of androgens and glucocorticoids later in prenatal development, and so its presence before incubation is thought to slowly decrease across prenatal development as other hormone levels in the eggs are elevated (Gil, 2008). The results from Experiment 1 confirm that bobwhite quail egg yolk progesterone levels steadily decline across prenatal development, and are not detectable after prenatal day 13 (see Figure 3).

Results from Experiment 2 suggest that prenatal auditory learning was modified in chicks that hatched from eggs with elevated prenatal progesterone compared to eggs that did not receive progesterone treatments. Embryos that had elevated levels of prenatal progesterone following progesterone-infused oil injections before incubation did not form a postnatal preference for a familiar call that was presented to them 24 hours before hatching; however they did prefer the novel call, although the preference was not found to be significant. 
We know from previous studies that bobwhite quail have the ability to form a postnatal preference for auditory stimuli presented 24 hours before hatching (Lickliter \& Hellewell, 1992). The results from the Lickliter \& Hellewell (1992) study were replicated in the current study. In addition, the current study suggests that prenatal hormone levels, including progesterone, can affect prenatal learning outcomes in bobwhite quail.

It was previously established in bobwhite quail that if testosterone is elevated prior to incubation in bobwhite quail yolk, chicks that hatched from eggs treated with testosterone prior to incubation displayed an enhanced preference to a bobwhite maternal call they were exposed to 24 hours before hatch over a novel maternal call (Bertin et al., 2009). Also, testosterone-treated eggs produced chicks that needed less time to learn a specific maternal call, and they showed an increased preference to the call in a postnatal simultaneous choice test. In particular, testosterone-treated chicks moved faster to the source of the call and stayed in the vicinity of the call longer than control groups (Bertin, et al., 2009).

Similar to the Bertin et al. (2009) study, it was demonstrated in the current study that elevation of prenatal androgens modifies postnatal phenotype in bobwhite quail hatchlings. Results from Experiment 2 indicate that bobwhite quail chicks that were treated with progesterone prior to incubation did not show postnatal preference for a bobwhite maternal call that was presented to them prenatally ( $24 \mathrm{hrs}$ before hatching). In both the 24 and $48 \mathrm{hr}$ age conditions, bobwhite quail chicks that hatched from progesterone treated eggs spent significantly less time in proximity to the familiarized maternal call compared to chicks hatched from eggs that were not treated with 
progesterone prior to incubation. Overall, the results from the 24-hr condition in Experiment 2 suggest that chicks hatched from P-treated eggs show a prenatal learning deficit compared to chicks hatched from eggs in the $\mathrm{V}$ and $\mathrm{C}$ groups. Both the vehicle and control groups demonstrated a significant postnatal preference for the familiarized call at $24 \mathrm{hr}$ of age in Experiment 2. In contrast, chicks from the $\mathrm{V}$ and $\mathrm{C}$ groups did not show a significant preference for the familiarized call when tested 48-hrs after hatching, as did the chicks from progesterone treated eggs. The lack of group differences was not surprising due to the difficulty of the test in the 48-hr prenatal condition; the familiar bobwhite maternal call was presented 24-hrs prior to hatch, and testing took place at 48hrs after hatching, which resulted in a 72-hr offset from the initial stimulus.

There is a potential explanation based on comparison studies in bobwhite quail that speaks to the deficit in prenatal auditory learning in chicks that hatched from Ptreated eggs. It is possible chicks that received elevated levels of progesterone prior to incubation experienced elevated prenatal arousal levels, which can interfere with prenatal auditory learning (Markham, Toth, \& Lickliter, 2006). Markham, Toth and Lickliter (2006) administered an intramuscular injection of norepinephrine, which elevated heart rate in bobwhite quail embryos. The embryos were then exposed to a variant of a maternal call, and tested for their preference for the familiar call over that of a novel maternal call. Embryos that received a norepinephrine injection prior to being exposed to a maternal call failed to form a preference for the familiar maternal call. In a separate experiment in the Markham et al. (2006) study, birds that were injected with norepinephrine prenatally were exposed to a familiar call after hatch, to see if perception was altered by the prenatal norepinephrine injection. Bobwhite chicks that were exposed 
to norepinephrine prenatally formed a preference for a maternal call presented after hatch, which strongly suggested that postnatal auditory perceptual processes were still in place. It is possible that progesterone injections prior to incubation somehow elevated prenatal arousal, potentially through loading pathways for the production of glucocorticoid stress hormones, thus interfering with prenatal perceptual learning. Follow-up studies are needed to examine this possibility.

Previous studies have demonstrated that bobwhite quail show the ability to form a preference for an individual maternal familiar call after passive exposure to that call for 120 minutes total over the course of 24 hours (Bertin et al., 2009). The original hypothesis in the present study was that chicks hatched from progesterone-treated eggs would not perform as well as vehicle and control chicks on the simultaneous choice test after 24 hours of passive exposure immediately following hatching between the familiarized call and a novel variant of the maternal assembly call (Call A or Call B).

Experiment 3 in the current study demonstrated that chicks hatched from progesterone-treated eggs showed a stronger preference for a familiar maternal call compared to chicks hatched from the $\mathrm{C}$ and $\mathrm{V}$ control groups when the call was presented $24 \mathrm{hr}$ postnatally in Experiment 3. In a previously mentioned study (Bertin et al., 2009), testosterone-treated bobwhite quail eggs were found to have an enhanced preference for a familiar call that was presented prenatally. Chicks spent more time in proximity to the familiar maternal call, and also approached the familiar call more quickly than vehicle or control groups (Bertin et al., 2009). In the present study, chicks that hatched from progesterone treated eggs also showed an enhanced preference for a maternal call that they were previously exposed to, but only did so when familiarized to the maternal call 
after hatching. It is possible that progesterone elevation in egg yolk prior to incubation leads to modification of testosterone levels in the egg yolk in later prenatal development. The mechanism by which progesterone augments postnatal perceptual learning in bobwhite quail chicks remains unclear.

Some investigators have suggested that bird mothers either passively or actively adjust hormone levels in egg yolk in order to provide a compensatory mechanism for their offspring in potentially adverse environmental conditions. A previous study in Japanese quail found a relationship between maternal stress and elevated progesterone in egg yolk (Guibert, Richard-Yris, Lumineau, Kotrschal, Bertin, Petton, \& Houdelier, 2011). Japanese quail hens that experienced mild and unpredictable stress during egg formation laid eggs with more yolk and albumen, as well as elevated levels of progesterone and testosterone compared to eggs laid by Japanese quail hens that did not experience stress during egg formation (Guibert et al., 2011). Chicks hatched from eggs laid by mothers who received mild prenatal stress were found to be more emotionally reactive. Although the findings from this study cannot be translated directly to bobwhite quail, they suggest a possible link between elevated stress during egg formation and subsequent progesterone elevation in egg yolk, which was the assumed link underlying the current study. Based on the assumption that elevated stress during egg formation results in elevated progesterone levels in the egg yolk, the findings from our current study suggest that prenatal progesterone elevation may act as a compensatory measure in adverse environmental conditions.

Based on evidence from a previous study in Japanese quail (Guibert et al., 2011), laying hens that are exposed to stressful stimuli produce eggs that have elevated yolk 
hormone concentrations, including elevated progesterone and testosterone levels, compared to non-stressed hens. As previously discussed, whether egg yolk hormone concentrations are actively or passively altered by laying hens remains unclear. Assumptions can be made from findings in the present study and other studies in birds that laying hens use yolk hormone concentrations as a compensatory mechanism for environmental challenges that their offspring may face after hatching. Based on this idea, bobwhite quail hens may use elevated prenatal progesterone concentrations in order to modify offspring phenotype in a way that increases fitness in an adverse environment. Although they are two different species, bobwhite quail and Japanese quail are physiologically similar, so it is not inconceivable that a similar relationship between elevated maternal stress and elevated yolk progesterone levels exists similar to the Guibert et al. (2011) study, however a comparative study in bobwhite quail is needed to verify the hypothesis that yolk hormone levels can vary with changes to maternal experience.

In sum, the present study presents evidence that maternal effects, especially the presence of maternally-derived hormones in pre-incubated egg yolk, can have a significant effect on offspring phenotype. Studies like the one presented here lay the foundation for future research that could seek to determine the origins of phenotypic plasticity via maternally derived hormones present in the prenatal environment of birds.

\section{Limitations}

There were a few limitations to the current study that will be discussed in the following section. First, I will highlight the limited amount of knowledge we have on the complex relationship between various compounds in the egg yolk (including both 
hormones and other organic compounds) and subsequent embryonic development and behavior. Second, I will outline limitations and constraints to rearing chicks in a lab environment without maternal demographic information or the ability to sex individual birds. Lastly, I will focus on the difficulty to generalize the findings of the present study to other species, particularly mammals.

\section{Complexity of non-genetic prenatal influences}

The study of the effects of maternal hormones on offspring phenotype is currently in its infancy, so it is difficult to speculate on the mechanisms that underlie changes to postnatal phenotype following elevation of progesterone in pre-incubated bobwhite quail yolk. Across prenatal development, the embryo is exposed to a wide variety of organic compounds, including hormones of maternal origin (Gil, 2003; 2008), endogenous hormones created by the embryo itself (von Engelhardt, Henriksen, \& Groothuis, 2009, environmental toxins such as mercury and pesticides (Peakall, 1985) and carotenoids that can vary based on maternal diet (Cucco, Guasco, Malacarne, \& Ottonelli, 2006). Any number of these aforementioned organic compounds, acting separately or together across prenatal development, has been found to affect postnatal behavior and physiology in birds. Moreover, variations in the deposition of yolk hormone levels across different egg yolk layers expose bird embryos to certain hormones at different time periods across prenatal development (Groothuis et al., 2008). So, it is not only the content of the egg yolk that can affect chick phenotype, but also the timing of exposure to organic compounds across prenatal development.

Although previous studies have demonstrated that postnatal phenotype can be altered following the manipulation of various organic compounds like hormones or 
carotenoids in the yolk of bird eggs, there is still much to be learned before any conclusions can be drawn in regards to specific mechanisms that underlie the effects elevated progesterone on prenatal and postnatal auditory learning.

\section{Unknown maternal effects}

Since bobwhite quail eggs used in the present study came from a commercial supplier, there was no information regarding the demographics of the mothers of the birds used in these experiments. As previously mentioned, many factors including maternal age (Okuliarova et al., 2009), exposure to stressful stimuli during egg formation (Guibert et al., 2011), and diet (Schwabl, 2006) could potentially affect hormone content and/or other chemicals in the egg yolk and subsequent offspring phenotype. Since this information could not be collected in the present study, it would be difficult to ascertain if there were any effects on offspring behavior due to indirect maternal effects.

\section{Sex effects}

It is very difficult in our lab to determine how elevated progesterone affects individual behavior and physiology across the lifespan due to the fact that subjects in the lab are only kept until 96 hours of age. It would be useful to know if elevated progesterone affects behavior and physiology at the age of reproduction, as this could represent a potential mechanism by which the behavior and physiology of future generations could be altered by way of variation of yolk steroid hormone content in a previous generation. Moreover, sex is nearly impossible to determine in bobwhite quail hatchlings without the aid of genetic tests in the days following hatching. Different levels of hormones present in the prenatal environment can adjust clutch sex ratios in birds (Krackow, 1995), and sex differences have been found in postnatal behavior and 
physiology following prenatal hormone exposure (Groothuis et al., 2008). It is currently unknown if progesterone elevation affects sex ratios in bobwhite quail, so future studies in bobwhite quail and other species of bird should address this question.

\section{Species generalization}

Lastly, it could be difficult to generalize these findings to other species. There is a great deal of variability in hormone content across different species of bird, which can depend on developmental and behavioral characteristics (precocial verses altricial birds). While elevated prenatal progesterone does affect postnatal phenotype in bobwhite quail, this may not be the case in other bird species. It is not uncommon to find conflicting results in the expression of phenotype following egg yolk hormone manipulations (Guibert et al., 2011). It is also difficult to generalize the findings from this study to mammals, including humans. One of the reasons why this is the case is that birds only have one chance to affect hormone content in the prenatal environment, while mammalian fetuses are exposed to varying levels of exogenous and endogenous hormones across prenatal development.

\section{Future directions}

Birds are a useful model for studying the effects of maternally derived hormones on offspring phenotype. Bobwhite quail develop very quickly and are hatched with highly developed sensory systems. Researchers that use birds to study the effects of elevated maternally-derived hormone levels on postnatal phenotype can produce a relatively large sample size in a shorter period of time compared to studies in mice and rats. In addition, bird eggs are free of influence from exogenous maternal and sibling 
hormones, which allows for a "cleaner" observation of the effects of maternally derived hormones on offspring phenotype.

Future studies could seek to determine the relationship between neural development in areas associated with learning, memory, and stress and the elevation of progesterone prior to incubation in order to link progesterone elevation before incubation to brain development. Due to the complex nature of brain/body/environment exchanges in prenatal development, it is difficult without including measures of neural growth and development to determine how the elevation of progesterone could have affected the growth and function of perceptual and memory systems.

Progesterone is only one of many compounds found in the egg yolk that have shown to cause changes to offspring phenotype. Future studies could manipulate other maternally-derived androgens, corticosterone concentrations later in development, and even carotenoid compounds. Carotenoids have been known to affect behavior and immunity in birds. Although there have been very few studies that have examined the effects of prenatal egg yolk carotenoid levels on postnatal behavior in birds, one study found that high concentrations of carotenoids and low concentrations of testosterone in grey partridge egg yolk can create maladaptive postnatal immune function and behavior (Cucco et al., 2006).

In addition, future studies should attempt to discover how maternal hormones are transferred to the egg, and which factors affect yolk hormone content and subsequent offspring behavior. The results from the current study do not speak directly to any of the hypotheses put forth by Groothuis and Schwabel (2008) attempted to explain the relationship between maternal behavior and physiology, and egg yolk steroid 
concentrations. Some recent work in chickens suggests that small changes to the environment can have effects on yolk hormone content and offspring behavior. Laying hens that were exposed to moderately higher than normal temperature levels produced eggs with significantly higher yolk concentrations of testosterone, estradiol, and progesterone compared to hens exposed to normal temperatures (Bertin et al., 2013). Offspring produced by laying hens that experienced elevated temperatures were less emotionally reactive than the offspring from control hens. This study thus provides some evidence for the physiological epiphenomenon hypothesis put forth by Groothuis and Schwabel (2008). Future studies that explore other environmental effects on laying hen behavior and subsequent progesterone content in the yolk of their eggs are needed to further our understanding of how maternal variation of progesterone can be adaptive when certain environmental conditions are present.

A final note can be made on the advantages of using birds to study the effects of maternally derived hormones on offspring phenotype. Bobwhite quail are an appropriate subject for a follow-up study on the lifelong effects of elevated prenatal progesterone (a non-genetic maternally derived influence), due to their relatively short lifespan (about 2 years). Although these previous studies in human have demonstrated that malnutrition and stress experienced by pregnant mothers contribute to maladaptive behavior and physiology in their offspring (Brown, Susser, Lin, Neugebauer, \& Gorman, 1995; Stein, Zybert, Van de Bor, \& Lumey, 2004; Franzek, Sprangers, Janssens, Van Duijn, \& Van De Wetering, 2008) the role of prenatal maternal hormone exposure to the fetus as the result of maternal stress is not currently understood. Future studies could experimentally elevate prenatal progesterone along with other hormones of maternal origin and then 
perform a variety of behavioral and physiological tests across several generations of bobwhite quail to test whether there are multi-generational effects of on offspring phenotype following hormonal manipulation. Unfortunately, studies such as this in birds would not provide specific information for the influence of maternally derived progesterone on postnatal behavior in humans. Nevertheless, studies that examine embryonic exposure to maternally derived hormones during prenatal development in birds are much less expensive and take considerably less time than comparative studies in humans. It is my hope that the findings from the current study could inform future research that investigates the influence of non-genetic maternal effects on offspring behavior and physiology. 


\section{REFERENCES}

Alberts, J. R., \& Cramer, C. P. (1988). Ecology and experience: Sources of means and meaning of developmental change. In E. M. Blass (Ed.), Handbook of behavioral neurobiology. Vol. 9. Developmental psychobiology and behavioral ecology (pp. 1-67). New York: Plenum Press.

Alberts, J. R. (2008). The nature of nurturant niches in ontogeny. Philosophical Psychology, 21(3), 295-303.

Almasi, B., Rettenbacher, S., Müller, C., Brill, S., Wagner, H., \& Jenni, L. (2012). Maternal corticosterone is transferred into the egg yolk. General and Comparative Endocrinology, 178(1), 139-144.

Badyaev, A.V., Schwabl, H., Young, R.L., Duckworth, R.A., Navara, K.J. \& Parlow, A.F. (2005). Adaptive sex differences in growth of pre-ovulation oocytes in a passerine bird. Proceedings of the Royal Society Biology, 272, 2165 -2172.

Bateson, P. P. G. (1966). The characteristics and context of imprinting. Biological Reviews, 41, 177-220.

Bateson, P. P. G. (1973). The imprinting of birds. In S. A. Barnett (Ed.), Ethology and development (pp. 1-15). Philadelphia: Lippincott.

Bertin, A., Chanson, M., Delaveau, J., Mercerand, F., Möstl, E., Calandreau, L., Arnould, C., Leterrier, C., \& Collin, A. (2013). Moderate Heat Challenge Increased Yolk Steroid Hormones and Shaped Offspring Growth and Behavior in Chickens. PloS One, 8(2), e57670.

Bertin, A., \& Richard-Yris, M. A. (2005). Mothering during early development influences subsequent emotional and social behaviour in Japanese quail. Journal of Experimental Zoology Part A: Comparative Experimental Biology,303(9), 792801 .

Bertin, A., Richard-Yris, M.A., Houdelier, Cecilia, Lumineau, S., Mostl, Er., Kuchar, A., Hirschenhauser, K., \& Kotrschal, K. (2008). Habituation to humans affects yolk steroid levels and offspring phenotype in quail. Hormones and Behavior, 54, 396402.

Bertin, A., Richard-Yris, M. A., Houdelier, C., Richard, S., Lumineau, S., Kotrschal, K., \& Mostl, E. (2009). Divergent selection for inherent fearfulness leads to divergent yolk steroid levels in quail. Behaviour, 146(6), 757-770. 
Bertin, A., Richard-Yris, M.-A., Mostl, E., \& Lickliter, R. (2009). Increased yolk testosterone facilitates prenatal perceptual learning in Northern bobwhite quail (Colinus virginianus). Hormones and Behavior, 56, 416-422.

Breuner, C. (2008). Maternal stress, glucocorticoids, and the maternal/fetal match hypothesis. Hormones and Behavior, 54, 485-487.

Breuner, C. W., \& Hahn, T. P. (2003). Integrating stress physiology, environmental change, and behavior in free-living sparrows. Hormones and Behavior, 43(1), 115-123.

Brown, A. S., Susser, E. S., Lin, S. P., Neugebauer, R., \& Gorman, J. M. (1995). Increased risk of affective disorders in males after second trimester prenatal exposure to the Dutch hunger winter of 1944-45. The British Journal of Psychiatry, 166(5), 601-606.

Buntin, J. D. (1996). Neural and hormonal control of parental behavior in birds. Advances in the Study of Behavior, 25, 161-213.

Cameron, N., Del Corpo, A., Diorio, J., McAllister, K., Sharma, S., \& Meaney, M. J. (2008). Maternal programming of sexual behavior and hypothalamic-pituitarygonadal function in the female rat. PloS One, 3(5), e2210.

Champagne, F. A., Francis, D. D., Mar, A., \& Meaney, M. J. (2003). Variations in maternal care in the rat as a mediating influence for the effects of environment on development. Physiology \& Behavior, 79(3), 359-371.

Chan, S. Y., Vasilopoulou, E., \& Kilby, M. D. (2009). The role of the placenta in thyroid hormone delivery to the fetus. Nature Reviews Endocrinology, 5, 45-54.

Clark, M. M., \& Galef, B. G. (1995). Prenatal influences on reproductive life history strategies. Trends in Ecology \& Evolution, 10, 151-153.

Daisley, J. N., Bromundt, V., Mostl, E., \& Kotrschal, K. (2005). Enhanced yolk testosterone influences behavioral phenotype independent of sex in Japanese quail chicks (Coturnix japaonica). Hormones and Behavior, 47, 185-194.

Dufty Jr, A. M., Clobert, J., \& Møller, A. P. (2002). Hormones, developmental plasticity and adaptation. Trends in Ecology \& Evolution, 17(4), 190-196.

Egger, G., Liang, G., Aparicio, A., \& Jones, P. A. (2004). Epigenetics in human disease and prospects for epigenetic therapy. Nature, 429, 457-463. 
Fifer, W. P., \& Moon, C. (1988). Auditory experience in the fetus. In W. P. Smotherman \& S. R. Robinson (Eds.), Behavior of the fetus (pp. 175-188). Caldwell, NJ: Telford Press.

Francis, D. D., Champagne, F. A., Liu, D., \& Meaney, M. J. (1999). Maternal care, gene expression, and the development of individual differences in stress reactivity. Annals of the New York Academy of Sciences, 896(1), 66-84.

Francis, D., Diorio, J., Liu, D., \& Meaney, M. J. (1999). Nongenomic transmission across generations of maternal behavior and stress responses in the rat. Science, 286(5442), 1155-1158.

Francis, D. D., \& Meaney, M. J. (1999). Maternal care and the development of stress responses. Current opinion in neurobiology, 9(1), 128-134.

Franzek, E. J., Sprangers, N., Janssens, A. C. J. W., Van Duijn, C. M., \& Van De Wetering, B. J. (2008). Prenatal exposure to the 1944-45 Dutch 'hunger winter'and addiction later in life. Addiction, 103(3), 433-438.

Gil, D. (2003). Golden eggs: Maternal manipulation of offspring phenotype by egg andgrogen in birds. Ardeola, 50, 281-294.

Gil, D. (2008). Hormones in avian eggs: physiology, ecology and behavior. Advances in the Study of Behavior, 38, 337-398.

Gottlieb, G. (1968). Prenatal behavior of birds. Quarterly Review of Biology, 148-174.

Gottlieb, G. (1971). Ontogenesis of sensory function in birds and mammals. In: The biopsychology of development (Tobach, E., Aronson, L. \& Shaw, E. Eds.) (pp. 67128). Academic, New York.

Gottlieb, G. (1983). Development of species identification in ducklings: Perceptual specificity in the wood duck embryo requires sib stimulation for maintenance. Developmental Psychobiology, 16(4), 323-333.

Gottlieb, G. (1997). Synthesizing nature-nurture: Prenatal roots of instinctive behavior. Psychology Press.

Gould, S. J. (1977). Ontogeny and Phylogeny. Belknap press.

Groothuis, G.G., Muller, W., von Engelhardt, N., Carere, C., \& Eising, C. (2005). Maternal hormones as a tool to adjust offspring phenotype in avian species. Neuroscience and Biobehavioral Reviews, 29, 329-352. 
Groothuis, T. G. G., \& Schwabl, H. (2002). Determinants of within- and among-clutch variation in levels of maternal hormones in Black-Headed Gull eggs. Functional Ecology, 16, 281-289.

Groothuis, T. G. G., \& Schwabl, H. (2008). Hormone-mediated maternal effects in birds: mechanisms matter but what do we know of them? Philosophical Transactions of The Royal Society Biological Sciences, 363, 1647-1661.

Guesdon, V., Bertin, A., Houdelier, C., Lumineau, S., Formanek, L., Kotrschal, K., Mostl, E., \& Richard-Yris, M. A. (2011). A place to hide in the home-cage decreases yolk androgen levels and offspring emotional reactivity in Japanese quail. PloS One, 6(9).

Guibert, F., Richard-Yris, M. A., Lumineau, S., Kotrschal, K., Guémené, D., Bertin, A., Mostl, E., \& Houdelier, C. (2010). Social instability in laying quail: consequences on yolk steroids and offspring's phenotype. Plos One, 5(11), e14069.

Guibert, F., Richard-Yris, M. A., Lumineau, S., Kotrschal, K., Bertin, A., Petton, C., \& Houdelier, C. (2011). Unpredictable mild stressors on laying females influence the composition of Japanese quail eggs and offspring's phenotype. Applied Animal Behaviour Science, 132(1), 51-60.

Harshaw, C., \& Lickliter, R. (2007). Interactive and vicarious acquisition of auditory preferences in Northern bobwhite (Colinus virginianus) chicks. Journal of Comparative Psychology, 121(3), 320.

Hackl, R., Bromundt., V., Daisley, J., Kotrschal, K., \& Mostl, E., (2003) Distribution and origin of steroid hormones in the yolk of Japanese quail eggs (Coturnix coturnix japonica). Journal of Comparative Physiology B, 173, 327-331.

Hayward, L. S., \& Wingfield, J. C. (2004). Maternal corticosterone is transferred to avian yolk and may alter offspring growth and adult phenotype. General and Comparative Endocrinology, 135, 365-371.

Hayward, L. S., Richardson, J. B., Grogan, M. N., \& Wingfield, J. C. (2006). Sex differences in the organizational effects of corticosterone in the egg yolk of quail. General and Comparative Endocrinology, 146(2), 144-148.

Henriksen, R., Rettenbacher, S., \& Groothuis, T. G. (2011). Prenatal stress in birds: Pathways, effects, function and perspectives. Neuroscience \& Biobehavioral Reviews, 35(7), 1484-1501.

Hess, E. H. (1962). Imprinting and the 'critical period' concept. In E. L. Bliss (Ed), Roots of behavior (pp. 254-273). Haffner, New York. 
Holliday, R. (2006). Epigenetics: a historical overview. Epigenetics, 1(2), 76-80.

Honeycutt, H. (2006). Studying Evolution in Action: Foundations for a Transgenerational Comparative Psychology. International Journal of Comparative Psychology, 19(2).

Honeycutt, H., \& Lickliter, R. (2001). Order-dependent timing of unimodal and multimodal stimulation affects prenatal auditory learning in bobwhite quail embryos. Developmental Psychobiology, 38, 1-10.

Honeycutt, H., \& Lickliter, R. (2002). Prenatal experience and postnatal perceptual preferences: Evidence for attentional-bias in bobwhite quail embryos (Colinus virginianus). Journal of Comparative Psychology, 116, 270-276.

Holmes, M.C., \& Seckl, J.R. (2007). Mechanisms of disease: Glucocorticoids, their placental metabolism and fetal 'programming' of adult pathophysiology. Nature Clinical Practice Endocrinology \& Metabolism, 3, 479-488.

Huang, E.S. \& Nalbandov, A.V. (1979) Steroidogenesis of chicken granulosa and theca cells: in vitro incubation system. Biology of Reproduction, 20, 442-453.

Katz, A., Oyama, R. K., Feng, N., Chen, X., \& Schlinger, B. A. (2010). 11ßhydroxysteroid dehydrogenase type 2 in zebra finch brain and peripheral tissues. General and Comparative Endocrinology, 166(3), 600-605.

Kitaysky, A. S., Piatt, J. F., \& Wingfield, J. C. (2007). Stress hormones link food availability and population processes in seabirds. Marine Ecology-Progress Series, 352, 245.

Klusoňová, P., Kučka, M., Ergang, P., Mikšík, I., Bryndová, J., \& Pácha, J. (2008). Cloning of chicken 11 $\beta$-hydroxysteroid dehydrogenase type 1 and its tissue distribution. The Journal of Steroid Biochemistry and Molecular Biology,111(3), 217-224.

Kuo, Z.Y. (1967). The dynamics of behavior development: An epigenetic view. New York: Random House.

Licht, P., Frank, L. G., Pavgi, S., Yalcinkaya, T. M., Siiteri, P. K. \& Glickman, S. E. (1992). Hormonal correlates of 'masculinization' in female spotted hyaenas (Crocuta crocuta). 2. Maternal and fetal steroids. Journal of Reproduction \& Fertility, 95, 463-474.

Lickliter, R., \& Hellewell, T. B. (1992). Contextual determinants of auditory learning in bobwhite quail embryos and hatchlings. Developmental Psychobiology, 25(1), 1731. 
Lickliter, R., \& Honeycutt, H. (2003). Developmental dynamics: toward a biologically plausible evolutionary psychology. Psychological Bulletin, 129(6), 819.

Lickliter, R., \& Schneider, S. M. (2006). Role of development in evolutionary change: A view from comparative psychology. International Journal of Comparative Psychology, 19, 150-167.

Mayr, E. (1982). The growth of biological thought: Diversity, evolution, and inheritance. Harvard University Press.

Markham, R. G., Toth, G., \& Lickliter, R. (2006). Prenatally elevated physiological arousal interferes with perceptual learning in bobwhite quail (Colinus virginianus) embryos. Behavioral neuroscience, 120 (6), 1315-1325.

Marshall, R.C., Leisler, B., Catchpole, C.K. \& Schwabl, H. (2005). Male song quality affects circulating but not yolk steroid concentrations in female canaries (Serinus canaria). Journal of Experimental Biology, 208, 4593-4598.

Mazuc, J., Bonneaud, C., Chastel, O. \& Sorci, G. (2003). Social environment affects female and egg testosterone levels in the house sparrow (Passer domesticus). Ecology Letters, 6, 1084-1090.

McEwen, B. S., \& Sapolsky, R. M. (1995). Stress and cognitive function. Current Opinion in Neurobiology, 5(2), 205-216.

Michel, G.F., \& Moore, C.L. (1995). Developmental psychobiology: An interdisciplinary science. Cambridge, Massachusetts: The MIT Press.

Mignon-Grasteau, S., Roussot, O., Delaby, C., Faure, J. M., Mills, A., Leterrier, C., ... \& Beaumont, C. (2003). Factorial correspondence analysis of fear-related behaviour traits in Japanese quail. Behavioural Processes, 61(1), 69-75.

Mostl, E., Spendier, H., \& Kotrschal, K. (2001). Concentration of immunoreactive progesterone and androgens in the yolk of hens' eggs (Gallus domesticus). Wiener Tierarztliche Monatsschrift, 88(3), 62-65.

Mousseau, T. A., \& Fox, C. W. (1998). The adaptive significance of maternal effects. Trends in Ecology \& Evolution, 13(10), 403-407.

Navara, K.J., Siefferman, L.M., Hill, G.E. \& Mendonça, M.T. (2006). Yolk androgens vary inversely to maternal androgens in Eastern Bluebirds: an experimental study. Functional Ecology, 20, 449-456. 
Okuliarová, M., Škrobánek, P., \& Zeman, M. (2009). Variability of yolk testosterone concentrations during the reproductive cycle of Japanese quail. Comparative Biochemistry and Physiology Part A: Molecular \& Integrative Physiology, 154(4), 530-534.

Perré, Y., Wauters, A. M., \& Richard-Yris, M. A. (2002). Influence of mothering on emotional and social reactivity of domestic pullets. Applied Animal Behaviour Science, 75(2), 133-146.

Porter, T.E., Hargis, B.M., Silsby, J.L. \& El Halawani, M.E. (1989). Differential steroid production between theca interna and theca externa cells: A three cell model for follicular steroidogenesis in avian species. Endocrinology, 125, 109-116.

Pilz, K.M., Smith, H.G., Sandell, M.I. \& Schwabl, H. (2003). Interfemale variation in egg yolk androgen allocation in the European starling: do high-quality females invest more? Animal Behaviour, 65, 841-850.

Plotsky, P. M., \& Meaney, M. J. (1993). Early, postnatal experience alters hypothalamic corticotropin-releasing factor (CRF) mRNA, median eminence CRF content and stress-induced release in adult rats. Molecular Brain Research, 18(3), 195-200.

Radell, P. L., \& Gottlieb, G. (1992). Developmental intersensory interference: Augmented prenatal sensory experience interferes with auditory learning in duck embryos. Developmental Psychology, 28(5), 795.

Rettenbacher, S., Mostl, E., Hackl, R., \& Palme, R. (2005). Corticosterone in chicken eggs. Annals of The New York Academy of Sciences, 1046, 193-203.

Reynolds, G. D., \& Lickliter, R. (2002). Effects of prenatal sensory stimulation on heart rate and behavioral measures of arousal in bobwhite quail embryos. Developmental Psychobiology, 41, 112-122.

Richard-Yris, M.A., Michel, N., \& Bertin, A. (2005). Nongenomic inheritance of emotional reactivity in Japanese quail. Developmental Psychobiology, 46, 1-12.

Romanoff, A. L., \& Romanoff, A. J. (1949). The Avian Egg. Ithaca: Cornell University.

Roth, T. L., Lubin, F. D., Funk, A. J., \& Sweatt, J. D. (2009). Lasting epigenetic influence of early-life adversity on the BDNF Gene. Biological psychiatry, 65(9), 760-769.

Royle, N. J., Surai, P. F., \& Hartley, I. R. (2001). Maternally derived androgens and antioxidants in bird eggs: complementary but opposing effects? Behavioral Ecology, 12(4), 381-385. 
Sarma, H.N., Manikkam, M., Herkimer, C., Dell'Orco, J., Welch, K.B., Foster, D.L., \& Padmanabhan, V. (2005). Fetal programming: Excess prenatal testosterone reduces postnatal luteinizing hormone, but not follicle stimulating hormone responsiveness to estradiol negative feedback in the female. Endocrinology, 146, 4281-4291.

Savage, J. S., Fisher, J. O., \& Birch, L. L. (2007). Parental influence on eating behavior: conception to adolescence. The Journal of Law, Medicine \& Ethics, 35, 22-34.

Schwabl, H. (1993). Yolk is a source of maternal testosterone for developing birds. Proceedings of the National Academy of Sciences of the United States of America, 90, 11446-11450.

Schwabl, H. (1996a). Environment modifies the testosterone levels of a female bird and its eggs. Journal of Experimental Zoology, 276, 157-163.

Shimmura, T., Kamimura, E., Azuma, T., Kansaku, N., Uetake, K., \& Tanaka, T. (2010). Effect of broody hens on behaviour of chicks. Applied Animal Behaviour Science, 126(3), 125-133.

Silverin, B. (1998). Behavioural and hormonal responses of the pied flycatcher to environmental stressors. Animal Behaviour, 55(6), 1411-1420.

Sockman, K. W. \& Schwabl, H. 2000 Yolk androgens reduce offspring survival. Proceedings of the Royal Academy of Sciences Biology, 267, 1451-1456.

Stein, A. D., Zybert, P. A., Van de Bor, M., \& Lumey, L. H. (2004). Intrauterine famine exposure and body proportions at birth: the Dutch Hunger Winter. International Journal of Epidemiology, 33(4), 831-836.

Tanvez A., Parisot M., Chastel O., Leboucher G. (2008). Does maternal social hierarchy affect yolk testosterone deposition in domesticated canaries? Animal Behaviour, 75, 929-934.

Verboven, N., Monaghan, P., Evans, D. M., Schwabl, H., Evans, N., Whitelaw, C., \& Nager, R. G. (2003). Maternal condition, yolk androgens and offspring performance: a supplemental feeding experiment in the lesser black-backed gull (Larus fuscus). Proceedings of the Royal Society of London. Series B: Biological Sciences, 270(1530), 2223-2232.

Wingfield, J. C., \& Kitaysky, A. S. (2002). Endocrine responses to unpredictable environmental events: stress or anti-stress hormones? Integrative and Comparative Biology, 42(3), 600-609. 
Weaver, I. C., Cervoni, N., Champagne, F. A., D'Alessio, A. C., Sharma, S., Seck1, J. R., Dymov, S., Szyf, M., \& Meaney, M. J. (2004). Epigenetic programming by maternal behavior. Nature Neuroscience, 7(8), 847-854.

Yang, Y., Shao, B., Zhang, J., Wu, Y., \& Ying, J. (2008). Analysis of eight free progestogens in eggs by matrix solid-phase dispersion extraction and very high pressure liquid chromatography with tandem mass spectrometry. Journal of Chromatography B, 870(2), 241-246. 
VITA

JOSHUA A. HERRINGTON

Born, Richmond, Virginia

2003-2007

B.S., Psychology

Virginia Tech

Blacksburg, Virginia

2007-2009

Research Associate, DePaul University

2009-2013

M.S., Psychology

Florida International University

Miami, Florida

$2013-2014$

Doctoral Candidate

Florida International University

Miami, Florida

\section{PUBLICATIONS AND PRESENTATIONS}

Herrington, J.A., Lickliter, R.L., \& Vallin, C. (submitted for review). Elevated Prenatal Progesterone Decreases Prenatal Learning and Elevates Postnatal Emotional Reactivity in Bobwhite Quail (Colinus Virginianus) Neonates.

Porter, N., Lerch, A., Jason, L.A., Sorenson, M., Fletcher, M.A., \& Herrington, J. (2010). A Comparison of immune functionality in viral versus non-viral CFS subtypes. Journal of Behavioral and Neuroscience Research, 8, 1-8.

Wright, D.B., \& Herrington, J.A. (2010) Problematic Standard Errors and Confidence Intervals for Skewness and Kurtosis. Behavior Research Methods, 43, 8-17.

Jason, L.A., Porter, N., Herrington, J., Sorenson, M., \& Kubow, S. (2009). Kindling and oxidative stress as contributors to myalgic encephalomyelitis/chronic fatigue syndrome. Journal of Behavioral and Neuroscience Research, 7, 1-17.

Jason, L.A., Timpo, P., Porter, N.S., Herrington, J.A., Brown, M., \& Torres-Harding, S., Friedberg, F. (2008). Activity logs as a measure of daily activity among patients with chronic fatigue syndrome. Journal of Mental Health, 18(6), 549-556.

Herrington, J.A. (2013). Elevation of Prenatal Progesterone Alters Phenotype in Bobwhite Quail Neonates: Implications for the Evolutionary Developmental Psychobiological Perspective. Presentation at the weekly Florida International University Center for Children and Families brown bag. 
Porter, N.S., Jason, L.A., Herrington, J.A., Sorenson, M. (2009). The Importance of Viral vs. Non-Viral Onset Subgrouping in an ME/CFS Community Sample: Differences in Cytokine Production and Expression. Oral presentation at the IACFS Conference in Reno, NV, April 2009.

Herrington, J.A., Vallin, C., \& Lickliter, R. (2013). Elevation of Prenatal Progesterone Enhances Postnatal Auditory Learning in Bobwhite Quail Neonates (Colinus Virginianus). Oral presentation at the ISDP Conference in San Diego, CA, October 2013.

J.Herrington, C. Vallin, and R. Lickliter. Elevated Prenatal Progesterone Increases Emotional Reactivity and Interferes with Prenatal Learning in Bobwhite Quail (Colinus virginianus). Poster Presentation at the ISDP Convention in New Orleans, LA. 2012.

J. Herrington and R. Lickliter. Efects of Elevated Prenatal Progesterone on Emotional Reactivity in Bobwhite Quail (Colinus virginianus) Neonates. Poster Presentation at the ISDP Convention in Washington, D.C. Nov 2011.

M. Bechor, J. Herrington, and R. Lickliter. Amount of Enriched Rearing Affects Bobwhite Quail Chicks Spatial Exploration of a Novel Environment. Poster Presentation at the ISDP Convention in San Diego, CA. Nov. 2010.

N.S. Porter, J.A. Herrington (2009). Activity Levels Affect Rate and Types of Play in Three Species of Non-Human Primates (Pan Pansicus, Pan Troglodytes, and Gorilla Gorilla). Poster Presentation at the ISDP Convention in Chicago, IL. Nov. 2009.

J.A. Herrington, N.S. Porter, A. Boulton (2009). Play Partner Preference in Three Species of Non-Human Primate: Comparisons of Play Behavior in Pan Pansicus, Pan Troglodytes, and Gorilla Gorilla Infants. Poster Presentation at the ISDP Convention in Chicago, IL. Nov. 2009.

Herrington, J., Porter, N., Jason, L., Sorensen, M., \& Lerch, A. (2009). Viral etiologies associated with immune activation in myalgic encephalomyelitis/chronic fatigue syndrome subtypes. Poster Presentation at the $81^{\text {st }}$ Annual Meeting of the Midwestern Psychological Association; Chicago, IL. 